\title{
SUMMABILITY OF HERMITE EXPANSIONS. II
}

\author{
S. THANGAVELU
}

\begin{abstract}
We study the summability of $n$-dimensional Hermite expansions where $n>1$. We prove that the critical index for the Riesz summability is $(n-1) / 2$. We also prove analogues of the Fejerr-Lebesgue theorem and Riemann's localisation principle when the index $\alpha$ of the Riesz means is $>$ $(3 n-2) / 6$.
\end{abstract}

\section{INTRODUCTION}

In this paper we study the summability of the $n$-dimensional Hermite expansions. Let $\varphi_{m}$ denote the $m$ th Hermite function. The $n$-dimensional Hermite functions $\Phi_{\nu}$ are defined as follows. For every multi-index $\nu=\left(\nu_{1}, \nu_{2}, \ldots, \nu_{n}\right)$ we set

$$
\Phi_{\nu}(x)=\varphi_{\nu_{1}}\left(x_{1}\right) \varphi_{\nu_{2}}\left(x_{2}\right) \cdots \varphi_{\nu_{n}}\left(x_{n}\right) .
$$

The functions $\Phi_{\nu}(x)$ are eigenfunctions of the operator $\left(-\Delta+|x|^{2}\right)$ with eigenvalues $(2|\nu|+n)$ where $\Delta$ is the $n$-dimensional Laplacian. The family $\left\{\Phi_{\nu}(x)\right\}$ defines an orthonormal system for $L^{2}\left(R^{n}\right)$. Given any $L^{p}$ function $f$ we can define its generalised Fourier coefficients by

$$
f^{\wedge}(\nu)=\int f(x) \Phi_{\nu}(x) d x .
$$

Thus to each function $f$ we have the associated Hermite expansion $f(x)=$ $\sum f^{\wedge}(\nu) \Phi_{\nu}(x)$ where the sum is extended over all multi-indices $\nu$. We can write the above series in the following way. For any nonnegative integer $k$ let us define the functions $\Phi_{k}(x, y)$ and operators $P_{k}$ by

$$
\begin{aligned}
& \Phi_{k}(x, y)=\sum_{|\nu|=k} \Phi_{\nu}(x) \Phi_{\nu}(y), \\
& P_{k} f(x)=\int \Phi_{k}(x, y) f(y) d y .
\end{aligned}
$$

Then $P_{k} f$ is the orthogonal projection of $f$ onto the subspace spanned by $\left\{\Phi_{\nu}(y):|\nu|=k\right\}$. The Hermite expansion then reads

$$
f(x)=\sum_{k \geq 0} P_{k} f(x) \text {. }
$$

Received by the editors September 1, 1987.

1980 Mathematics Subject Classification (1985 Revision). Primary 42C10; Secondary 42C15.

Key words and phrases. Hermite expansion, summability, Riesz means, oscillatory integrals. 
Since we are interested in the summability of the above series we define the Riesz and Cesàro means of the expansion as in the one-dimensional case. For $\alpha>0$ and $R>0$ the $R$ th Riesz means of order $\alpha$ is defined by

$$
S_{R}(\alpha) f(x)=\sum_{K \leq R}\left(1-\frac{K}{R}\right)^{\alpha} P_{k} f(x)
$$

where $K=2 k+n$. The $R$ th. Cesàro means of order $\alpha$ are defined by

$$
C_{R}(\alpha) f(x)=\left(A_{R}(\alpha)\right)^{-1} \sum_{k \leq R} A_{R-k}(\alpha) P_{k} f(x)
$$

where $A_{k}(\alpha)=\Gamma(k+\alpha+1) / \Gamma(k+1) \Gamma(\alpha+1)$. We are interested in the convergence of the above means to the function as $R$ tends to infinity.

This problem was considered by Hulanicki and Jenkins in [1]. They proved that for large values of $\alpha$ the Riesz means converge to the function in the norm. They studied the summability of eigenfunction expansions on a nilmanifold and deduced the summability results for the Hermite series as a corollary. We studied the summability of the one-dimensional Hermite expansions in [3]. There it is proved that $1 / 6$ is the critical index for the Riesz summability. In analogy with the one-dimensional case one expects that the critical index will be $(3 n-2) / 6$. But to our great surprise the critical index turns out to be $(n-1) / 2$ for $n>1$. The following theorem is the main result of this paper.

Theorem 1. Assume that $n \geq 2, \alpha>(n-1) / 2$ and $f$ is in $L^{p}, 1 \leq p \leq \infty$. Then the Riesz means $S_{R}(\alpha) f$ converges to $f$ in the norm as $R$ tends to infinity. If $\alpha<(n-1) / 2$, then there is an $L^{1}$ function $f$ for which the Riesz means $S_{R}(\alpha) f$ will not converge in the norm.

Thus we see that the behaviour of the $n$-dimensional Hermite series is more or less similar to the behaviour of the corresponding Fourier series when $n \geq 2$. This distinction between the one dimensional and the higher dimensions is explained to some extent by the behaviour of certain oscillatory integrals as we will see later. The estimates we are going to obtain for the Riesz kernel are not very good when $(3 n-2) / 6>\alpha>(n-1) / 2$. But for $\alpha>(3 n-2) / 6$ the estimates are quite neat and as in the one-dimensional case we can prove the Fejér-Lebesgue theorem and Riemann's localisation principle.

Theorem 2. Assume that $n \geq 2, \alpha>(3 n-2) / 6$ and $f$ is in $L^{p}, 1 \leq p \leq \infty$. If both $x$ and $-x$ are Lebesgue points for $f$ then $S_{R}(\alpha) f(x)$ converges to $f(x)$ as $R$ tends to infinity.

Theorem 3. Assume that $n \geq 2, \alpha>(3 n-2) / 6$ and $f$ is in $L^{p}, 1 \leq p \leq \infty$. If $f$ vanishes near the points $x$ and $-x$ then $S_{R}(\alpha) f(x)$ converges to 0 as $R$ tends to infinity.

This paper is organised as follows. In the next section we show that $\alpha>$ $(n-1) / 2$ is a necessary condition for the convergence of $S_{R}(\alpha) f$ for $L^{1}$ functions. In the third section we get an expression for the Riesz kernel in terms 
of an oscillatory integral and estimate the same when $\alpha>(3 n-2) / 6$. The estimation of the kernel when $\alpha>(n-1) / 2$ will be taken up in the fourth section. All these estimates are carried out for the three-dimensional case. Finally we indicate how to estimate the kernel in the general case. Once the kernel is estimated we can prove all the theorems as in the one-dimensional case. We refer the reader to [3].

This paper represents a part of my thesis written under the guidance of Professor E. M. Stein. It is a pleasure to thank my advisor for suggesting this problem and for the constant encouragement and many helpful suggestions he gave me during the course of this work.

\section{THE CRITICAL INDEX FOR THE RIESZ SUMMABILITY}

In this section we will show that if $\alpha<(n-1) / 2$ then there exists an $L^{1}$ function $f$ such that its Cesàro means will not converge to it. Since the Riesz and Cesàro means have identical behaviour this will prove that the Riesz means of order $\alpha<(n-1) / 2$ is not effective for $L^{1}$ functions. For the sake of simplicity we assume that $n=3$. The arguments can be carried out for any dimensions without much difficulty.

Suppose that the Cesàro means of order $\alpha$ of an $L^{1}$ function $f$ converges to $f$. Let $S_{n} f$ denote the partial sums of the expansion defined by

$$
S_{n} f(x)=\sum_{k \leq n} P_{k} f(x) .
$$

Proceeding as in the case of the one-dimensional case (see [3]) we can show that with $N=2 n+3$

$$
\left\|P_{n} f(x)\right\|_{1} \leq C N^{\alpha}\|f\|_{1} .
$$

Recall that the projections $P_{n}$ are given by

$$
P_{n} f(x)=\int \Phi_{n}(x, y) f(y) d y \text {. }
$$

From (2.1) and (2.3) we immediately obtain

$$
\sup _{x} \int\left|\Phi_{n}(x, y)\right| d y \leq C N^{\alpha} \text {. }
$$

We will prove the following theorem.

Theorem 2.1. There is a constant $C$ such that for large $n$ we have the estimate

$$
\sup _{y} \int\left|\Phi_{n}(x, y)\right| d x \geq C N \text {. }
$$

Consequently, for some $L^{1}$ function $f$ the Cesàro means of order $\alpha, \alpha<1$, will not converge.

To prove the theorem we need a good expression for the functions $\Phi_{n}(x, y)$. We are going to express $\Phi_{n}(x, y)$ in terms of the Mehler kernel $M_{z}(x, y)$. This 
kernel is defined for $|z|<1$ by $M_{z}(x, y)=\sum z^{n} \Phi_{n}(x, y)$. Since $M_{z}(x, y)$ is obtained by multiplying three copies of the one-dimensional Mehler kernel, we have the following formula:

$$
\begin{gathered}
M_{z}(x, y)=\pi^{-3 / 2}\left(1-z^{2}\right)^{-3 / 2} \exp \left\{B_{z}(x, y)\right\}, \\
B_{z}(x, y)=-\frac{1}{2} a^{2}\left(1+z^{2}\right) /\left(1-z^{2}\right)+2 b z /\left(1-z^{2}\right)
\end{gathered}
$$

where $a^{2}=\left(|x|^{2}+|y|^{2}\right)$ and $b=x_{1} y_{1}+x_{2} y_{2}+x_{3} y_{3}$. Let $G_{r}(t, x, y)=M_{z}(x, y)$ where $z=r e^{-2 i t}$. Then we have the following expression for $\Phi_{n}(x, y)$ :

$$
r^{n} \Phi_{n}(x, y)=\frac{1}{\pi i} \int_{0}^{\pi} e^{2 n i t} G_{r}(t, x, y) d t .
$$

With the notations $A_{r}(t)=\left(e^{2 i t}-r^{2} e^{-2 i t}\right)$ and

$$
B_{r}(t)=\left\{-\frac{1}{2} a^{2}\left(1+r^{2} e^{-4 i t}\right)+2 b r e^{-2 i t}\right\} /\left(1-r^{2} e^{-4 i t}\right)
$$

we can express $\Phi_{n}(x, y)$ in the following form:

$$
\Phi_{n}(x, y)=\lim _{r \rightarrow 1}\left(-i \pi^{-5 / 2}\right) \int_{0}^{\pi} e^{i N t} A_{r}(t)^{-3 / 2} e^{B_{r}(t)} d t .
$$

Let us set $\varphi(t)=-2 b \operatorname{cosec} 2 t+\frac{1}{2} a^{2} \cot 2 t$ and define the following three integrals:

$$
\begin{gathered}
F_{n}(x, y)=N \int(\sin 2 t)^{1 / 2}\left\{a^{2}-2 b \cos 2 t\right\}^{-1} e^{i N t} e^{i \varphi(t)} d t, \\
G_{n}(x, y)=\int(\sin 2 t)^{-1 / 2} \cos 2 t\left\{a^{2}-2 b \cos 2 t\right\}^{-1} e^{i N t} e^{i \varphi(t)} d t, \\
H_{n}(x, y)=\int(\sin 2 t)^{-3 / 2} \varphi^{\prime \prime}(t)\left\{\varphi^{\prime}(t)\right\}^{-2} e^{i N t} e^{i \varphi(t)} d t .
\end{gathered}
$$

All three integrals are extended from 0 to $\pi$. We are now ready to prove the following lemma.

Lemma 2.1. Given $x$ and $y$ such that $|x-y|>0$ and $|x+y|>0$ we have the expression

$$
\Phi_{n}(x, y)=C_{1} e^{i \pi / 4} F_{n}(x, y)+C_{2} G_{n}(x, y)+C_{3} H_{n}(x, y)
$$

where $C_{i}$ are constants, $C_{1}$ is real and $F_{n}, G_{n}$ and $H_{n}$ are the integrals defined above.

Proof. We are going to pass to the limit in the equation defining $\Phi_{n}(x, y)$. As $r$ tends to 1 , we see that $A_{r}(t)$ tends to $\sin 2 t$ and so at first sight it looks as though we cannot take the limit under the integral sign. But things are not so bad as we will see shortly. 
Let us write $C_{r}(t)=\left(e^{2 i t}+r^{2} e^{-2 i t}\right), D_{r}(t)=\left(r^{-1} e^{2 i t}-r e^{-2 i t}\right)$ and $E_{r}(t)=$ $\left(a^{2}-b r e^{-2 i t}-b r^{-1} e^{2 i t}\right)$. A simple calculation shows that $A_{r}^{\prime}(t)=2 i C_{r}(t)$, $B_{r}^{\prime}(t)=4 i r^{2} A_{r}(t)^{-2} E_{r}(t)$ so that

$$
A_{r}(t)^{-3 / 2}\left\{B_{r}^{\prime}(t)\right\}^{-1}=\left(4 i r^{2}\right)^{-1} A_{r}(t)^{1 / 2}\left(E_{r}(t)\right)^{-1} .
$$

Now,

$$
\begin{aligned}
\frac{d}{d t}\left\{A_{r}(t)^{-3 / 2}\left\{B_{r}^{\prime}(t)\right\}^{-1}\right\}= & \left(4 r^{2}\right)^{-1} A_{r}(t)^{-1 / 2} C_{r}(t)\left(E_{r}(t)\right)^{-1} \\
& +\left(2 r^{2}\right)^{-1} b A_{r}(t)^{1 / 2} D_{r}(t)\left(E_{r}(t)\right)^{-2} .
\end{aligned}
$$

Therefore, we can write

$$
\begin{aligned}
A_{r}(t)^{-3 / 2} \exp B_{r}(t)= & \partial_{t}\left\{A_{r}(t)^{-3 / 2} \exp B_{r}(t) / B_{r}^{\prime}(t)\right\} \\
& -\frac{1}{4} r^{-2} A_{r}(t)^{-1 / 2} \exp B_{r}(t) C_{r}(t) E_{r}(t)^{-1} \\
& -\frac{1}{2} r^{-2} b A_{r}(t)^{1 / 2} D_{r}(t) \exp B_{r}(t) E_{r}(t)^{-2} .
\end{aligned}
$$

In view of this equation we have

$$
\begin{gathered}
\int e^{i N t} A_{r}(t)^{-3 / 2} \exp \left\{B_{r}(t)\right\} d t=F_{r, n}(x, y)-G_{r, n}(x, y)-H_{r, n}(x, y), \\
F_{r, n}(x, y)=\int e^{i N t} \frac{d}{d t}\left\{A_{r}(t)^{-3 / 2} \exp B_{r}(t)\left(B_{r}^{\prime}(t)\right)^{-1}\right\} d t \\
G_{r, n}(x, y)=\left(4 r^{2}\right)^{-1} \int e^{i N t} \exp \left\{B_{r}(t)\right\} A_{r}(t)^{-1 / 2} C_{r}(t)\left(E_{r}(t)\right)^{-1} d t \\
H_{r, n}(x, y)=\left(2 r^{2}\right)^{-1} b \int e^{i N t} \exp \left\{B_{r}(t)\right\} A_{r}(t)^{1 / 2} D_{r}(t)\left(E_{r}(t)\right)^{-2} d t
\end{gathered}
$$

Now we need certain estimates for $A_{r}, B_{r}$ and $E_{r}$. The following estimates are easily obtained:

$$
\left|A_{r}(t)\right| \geq c \sin 2 t, \quad\left|\exp \left\{B_{r}(t)\right\}\right| \leq 1 \quad \text { and } \quad\left|E_{1}(t)\right| \geq\left(a^{2} \pm 2 b\right) .
$$

Since we are assuming that $\left(a^{2} \pm 2 b\right)>0$, we can find an $r_{0}=r_{0}(x, y)$ such that for $r_{0} \leq r \leq 1$ the lower bound $\left|E_{r}(t)\right| \geq \frac{1}{2}\left(a^{2} \pm 2 b\right)$ holds. With these estimates it is clear that we can pass to the limit under the integral sign in (2.13) and (2.14) getting the terms $G_{n}(x, y)$ and $H_{n}(x, y)$. In $F_{r, n}(x, y)$ we first integrate by parts and then pass to the limit. Noting that the boundary terms tend to 0 we obtain $F_{n}(x, y)$. This completes the proof of the lemma.

We now proceed to get estimates for the $L^{1}$ norm of $\Phi_{n}(x, y)$. Upper bounds for the $L^{1}$ norms of the terms $G_{n}(x, y)$ and $H_{n}(x, y)$ can be easily obtained. We have the following lemma. 
Lemma 2.2. There is a constant $C$ independent of $y$ and $n$ such that

$$
\int\left|G_{n}(x, y)\right| d x \leq C N^{1 / 2} \text { and } \int\left|H_{n}(x, y)\right| d x \leq C N^{1 / 2} .
$$

Proof. We will prove the estimate for $G_{n}(x, y)$. Estimating $H_{n}(x, y)$ is similar. Replacing $x$ and $y$ by $N^{1 / 2} x$ and $N^{1 / 2} y$ we need to show that

$$
\int\left|g_{n}(x, y)\right| d x \leq C
$$

where $g_{n}(x, y)$ is given by the integral

$$
g_{n}(x, y)=\int(\sin 2 t)^{-1 / 2} \cos 2 t\left\{a^{2}-2 b \cos 2 t\right\}^{-1} e^{i N \psi(t)} d t .
$$

Estimate (2.17) will follow once we show that the following estimate holds for $g_{n}$ :

$$
\left|g_{n}(x, y)\right| \leq C\left\{|x-y|^{-2}(1+|x-y|)^{-2}+|x+y|^{-2}(1+|x+y|)^{-2}\right\} .
$$

Splitting $g_{n}$ into four parts it is easy to see that we need to consider only the integral

$$
I=\int_{0}^{\pi / 4}(\sin 2 t)^{-1 / 2} \cos 2 t\left(a^{2}-2 b \cos 2 t\right)^{-1} e^{i N\{t+\varphi(t)\}} d t .
$$

For this integral it is immediate that $|I| \leq C|x-y|^{-2}$. The following estimates are easily checked:

$$
\left|\varphi^{\prime}(t) \sin ^{2} 2 t\right| \geq \frac{1}{2}|x-y|^{2}, \quad\left|\varphi^{\prime \prime}(t) \sin 2 t\right| \leq 4\left|\varphi^{\prime}(t)\right| .
$$

Integrating by parts and using the above estimates we obtain $\left|I_{1}\right| \leq C|x-y|^{-4}$. Combining the two estimates we get the required estimate.

To prove Theorem 2.1 we have to show that there is a $y_{0}$ such that

$$
\int\left|F_{n}\left(x, y_{0}\right)\right| d x \geq C N .
$$

Since there is rotational symmetry, we can assume that $y=\left(y_{1}, 0,0\right)$. We are going to apply the method of stationary phase to the integral defining $F_{n}$. We restrict ourselves to the region $x_{1} \geq 0, y_{1} \geq 0$, and $|x|^{2}+|y|^{2} \leq N$. Consider the function $\psi(t)=N t-b \operatorname{cosec} 2 t+\frac{1}{2} a^{2} \cot 2 t$. In the interval $0 \leq t \leq \pi / 4$ there is only one stationary point $t_{1}$ for the function $\psi$ given by $\cos 2 t_{1}=N^{-1} b+m$, where $m$ is defined by $m^{2}=1-N^{-1} a^{2}+N^{-2} b^{2}$. Likewise, for the function $\psi^{*}(t)=N t+b \operatorname{cosec} 2 t+\frac{1}{2} a^{2} \cot 2 t$ there is only one stationary point $t_{2}$ given by $\cos 2 t_{2}=-N^{-1} b+m$. Let us write $f(t)=(\sin 2 t)^{-1}\{\cos \psi(t)-\sin \psi(t)\}$ and $f^{*}(t)=(\sin 2 t)^{-1}\left\{\cos \psi^{*}(t)-\sin \psi^{*}(t)\right\}$. With this notation we can now prove the following lemma.

Lemma 2.3. Assume that $x_{1}, y_{1} \geq 0$, and $|x|^{2}+|y|^{2} \leq N$. Then we have, with some constant $c$,

$$
\operatorname{Re} F_{n}(x, y)=c N^{-1 / 2} m^{-1 / 2}\left\{f\left(t_{1}\right)+(-1)^{n+1} f^{*}\left(t_{2}\right)\right\}+O\left(N^{-1}\right) .
$$


Proof. To make the calculations easy let us replace $x$ and $y$ by $N^{1 / 2} x$ and $N^{1 / 2} y$, respectively and consider the following integral:

$$
I=\int_{0}^{\pi}(\sin 2 t)^{1 / 2}\left\{a^{2}-2 b \cos 2 t\right\}^{-1} e^{i N \rho(t)} d t .
$$

We write this integral as the sum of four integrals $I=I_{1}+(i)^{N} I_{2}+(i)^{N+1} I_{3}+$ $(i)^{3} I_{4}$ where $I_{4}=I_{1}, I_{3}=I_{2}$ and $I_{2}$ and $I_{1}$ are defined as follows:

$$
\begin{aligned}
& I_{1}=\int_{0}^{\pi / 4}(\sin 2 t)^{1 / 2}\left\{a^{2}-2 b \cos 2 t\right\}^{-1} e^{i N \rho(t)} d t, \\
& I_{2}=\int_{0}^{\pi / 4}(\sin 2 t)^{1 / 2}\left\{a^{2}-2 b \cos 2 t\right\}^{-1} e^{i N \rho^{*}(t)} d t .
\end{aligned}
$$

In the above the functions $\rho$ and $\rho^{*}$ are the functions given by $\rho(t)=t-$ $b \operatorname{cosec} 2 t+\frac{1}{2} a^{2} \cot 2 t$ and $\rho^{*}(t)=t+b \operatorname{cosec} 2 t+\frac{1}{2} a^{2} \cot 2 t$. Applying the method of stationary phase to the integral $I_{1}$ we get the following expression:

$$
I_{1}=(2 \pi)^{1 / 2} N^{-1 / 2} m^{-1 / 2}\left(\sin 2 t_{1}\right)^{-1} \exp \left\{i N \rho\left(t_{1}\right)+\pi / 4\right\}+O\left(N^{-1}\right) .
$$

Since $I_{4}=I_{1}$ we obtain after going back to the original variables

$$
\operatorname{Re}\left\{e^{i \pi / 4}\left(I_{1}-i I_{4}\right)\right\}=c N^{-1 / 2} m^{-1 / 2} f\left(t_{1}\right)+O\left(N^{-1}\right) .
$$

Similarly for the other two integrals we obtain

$$
\operatorname{Re}\left\{e^{i \pi / 4}(i)^{N}\left(I_{2}+i I_{3}\right)\right\}=c(-1)^{n+1} N^{-1 / 2} m^{-1 / 2} f^{*}\left(t_{2}\right)+O\left(N^{-1}\right) .
$$

This completes the proof of the lemma.

We now consider the main term in the expression of $\operatorname{Re} F_{n}(x, y)$. The first term has the lower bound

$$
\left|N^{-1 / 2} m^{-1 / 2} f\left(t_{1}\right)\right| \geq \frac{1}{2} N^{-1 / 2} m^{-1 / 2}\left(\sin 2 t_{1}\right)^{-1}\left|\cos 2 \psi\left(t_{1}\right)\right|
$$

and the second term is bounded by $2 N^{-1 / 2} m^{-1 / 2}\left(\sin 2 t_{2}\right)^{-1}$. Next we proceed to get a lower bound for $\left|\cos 2 \psi\left(t_{1}\right)\right|$. Also we need an estimate for the ratio of $\sin 2 t_{1}$ and $\sin 2 t_{2}$. To estimate the cosine term we use the following lemma due to Muckenhoupt [2].

Lemma 2.4 (Muckenhoupt). Let $L$ be an integer greater than 20 and let $I$ be a set of $L$ consecutive integers. If for $k$ in $I, \frac{1}{3} L \leq g(k+1)-g(k) \leq \pi / 4$ and $g(k+1)-g(k)$ is monotone decreasing in $k$, then for at least $2 / 3$ of the integers $k$ in $I$ we have $|\cos g(k)| \geq 1 /(200)$.

Proof. The intervals in which $|\cos x|<1 /(200)$ have length $<2 \sin ^{-1} 1 /(200)$ which is less than $1 /(90)$. The last of these intervals that contains any of the $g(k)$ 's then contains at most $[L / 30]+1$ of them where [ ] denotes the greatest integer. Before this each interval where $|\cos x|$ is greater than or equal to $1 /(200)$ will contain at least three times as many $g(k)$ 's as the preceding interval where $|\cos x|<1 /(200)$ because of the upper bound on $g(k+1)-g(k)$ 
and its monotonicity. Then for at most $[L / 30]+1+\frac{1}{4}\{L-[L / 30]-1\}$ of the $k$ 's in $I$ is $|\cos g(k)|<1 / 200$. Since $\frac{1}{L}[L / 30] \leq 1 / 30$, this number is bounded by $L(1 / 40+1 / 4+3 / 4 L)$ which is less than $\frac{1}{3} L$ since $L>20$. This completes the proof of the lemma.

Lemma 2.5. Let $\varepsilon=(2500)^{-1}$ and assume that $y_{1}^{2} \geq 4(2500)^{3}$. Assume that $y_{1}$ and $x_{1}$ satisfy the following three conditions:

$$
\begin{gathered}
y_{1}^{2} \leq x_{1}^{2} \leq y_{1}^{2}+\frac{1}{2} \varepsilon^{2} y_{1}^{2}, \\
\frac{1}{2} N\left(1-\varepsilon^{2}\right) \leq y_{1}^{2} \leq \frac{1}{2} N\left(1-\frac{1}{2} \varepsilon^{2}\right), \\
\frac{1}{4}\left(1-\varepsilon^{2}\right)^{-1} \varepsilon^{2} y_{1}^{2} \leq r^{2} \leq \frac{1}{2}\left(1-\frac{1}{2} \varepsilon^{2}\right)^{-1} \varepsilon^{2} y_{1}^{2},
\end{gathered}
$$

where we have put $r=\left|x^{\prime}\right|$. Let $I\left(y_{1}\right)$ denote the set of all consecutive integers $N$ satisfying

$$
2\left(1-\frac{1}{2} \varepsilon^{2}\right)^{-1} y_{1}^{2} \leq N \leq 2\left(1-\varepsilon^{2}\right)^{-1} y_{1}^{2} .
$$

Then for at least $2 / 3$ of the integers $N$ in $I\left(y_{1}\right)$ we have $\left|\cos 2 \psi\left(t_{1}\right)\right|>1 / 200$ where $t_{1}$ is the point defined in Lemma 2.3 .

Proof. We want to check the conditions of Lemma 2.4. Observe that under the hypothesis of the lemma we have $|x|^{2}+|y|^{2} \leq N$. Let us set $f(N)=$ $b / N+\left\{1-a^{2} / N+b^{2} / N^{2}\right\}^{1 / 2}$. It is easy to check that $f^{\prime}(N)>0$ and hence $f$ is an increasing function of $N$. Let $u=N^{-1 / 2} x$ and $v=N^{-1 / 2} y$ so that $f(N)=\sigma+\mu$ where $\sigma=u_{1} v_{1}, \tau^{2}=u_{1}^{2}+v_{1}^{2}$ and $\mu^{2}=1-\tau^{2}+\sigma^{2}$. We then have

$$
\begin{gathered}
v_{1}^{2} \leq u_{1}^{2} \leq v_{1}^{2}+\frac{1}{2} \varepsilon^{2} v_{1}^{2}, \\
\frac{1}{2}\left(1-\varepsilon^{2}\right) \leq v_{1}^{2} \leq \frac{1}{2}\left(1-\frac{1}{2} \varepsilon^{2}\right), \\
\frac{1}{4}\left(1-\varepsilon^{2}\right)^{-1} \varepsilon^{2} v_{1}^{2} \leq \rho^{2} \leq \frac{1}{2}\left(1-\frac{1}{2} \varepsilon^{2}\right)^{-1} \varepsilon^{2} v_{1}^{2},
\end{gathered}
$$

where $\rho=\left|u^{\prime}\right|$. From these it is clear that $2 \sigma \geq\left(1-\varepsilon^{2}\right)$ and $1-\tau^{2} \leq \varepsilon^{2}$. Therefore,

$$
f(N)=\sigma+\mu \geq 2 \sigma \geq\left(1-\varepsilon^{2}\right) \geq 2^{-1 / 2} .
$$

As $\sin ^{2} 2 t_{1}=\tau^{2}-2 \sigma \cos 2 t_{1} \geq|u-v|^{2} \geq \rho^{2} \geq \frac{1}{8} \varepsilon^{2}$, we have $\cos ^{2} 2 t_{1} \leq$ $\left(1-\frac{1}{8} \varepsilon^{2}\right) \leq\left(1-\frac{1}{16} \varepsilon^{2}\right)^{2}$ and hence $f(N) \leq\left(1-\frac{1}{16} \varepsilon^{2}\right)$. Thus we have $2^{-1 / 2} \leq$ $f(N) \leq\left(1-\frac{1}{16} \varepsilon^{2}\right)$. Since arc cos is decreasing in the interval $\left(2^{-1 / 2}, 1\right)$ and $f(N)$ is increasing $\cos ^{-1} f(N)$ is a decreasing function of $N$. Further we observe that if $\cos u \leq 1-\frac{1}{16} \varepsilon^{2}$, then $\left(1-\frac{1}{2} u^{2}\right) \leq\left(1-\frac{1}{16} \varepsilon^{2}\right)$ or $u \geq 8^{-1 / 2} \varepsilon$. Therefore, $\cos ^{-1} f(N) \geq 8^{-1 / 2} \varepsilon$. The condition $2^{-1 / 2} \leq f(N) \leq\left(1-\frac{1}{16} \varepsilon^{2}\right)$ translates into the inequality $8^{-1 / 2} \varepsilon \leq \cos ^{-1} f(N) \leq \pi / 4$. 
Let $L$ denote the number of consecutive integers $N$ in the interval $I\left(y_{1}\right)$. Clearly $L \geq \frac{1}{2} \varepsilon^{2} y_{1}^{2}$. As $\varepsilon^{3} y_{1}^{2} \geq 4$ we have $L \geq 2 \varepsilon^{-1}$ or $\varepsilon / 2 \geq 1 / L$. Thus we have $1 / L \leq \cos ^{-1} f(N) \leq \pi / 4$. Now we are in a position to check the conditions of the lemma. Let $g(N)=2 \psi\left(t_{1}\right)$, and $\cos ^{-1} f(N)=2 t_{1}$. Since $\psi^{\prime}\left(t_{1}\right)=0$ an easy calculation shows that $g^{\prime}(N)=2 t_{1}=\cos ^{-1} f(N)$. Therefore, the estimate $1 / L \leq(g(N+1)-g(N)) \leq \pi / 4$ is valid. By applying the lemma of Muckenhoupt we get $\left|\cos 2 \psi\left(t_{1}\right)\right|>1 /(200)$ for at least $2 / 3$ of the integers in $I\left(y_{1}\right)$.

Lemma 2.6. Let $t_{1}$ and $t_{2}$ be defined as in Lemma 2.3. Under the assumptions of Lemma 2.5

$$
\sin 2 t_{2} \geq 1600 \sin 2 t_{1} \text {. }
$$

Proof. Following the same notations as in Lemma 2.5 we have $\cos 2 t_{1}=\sigma+\mu$ and $\cos 2 t_{2}=\sigma-\mu$. Since $u_{1}^{2} \geq \frac{1}{2}\left(1-\varepsilon^{2}\right)$ and $v_{1}^{2} \geq \frac{1}{2}\left(1-\varepsilon^{2}\right)$ we have $4 u_{1}^{2} v_{1}^{2} \geq\left(1-\varepsilon^{2}\right)^{2}$ or $2 \sigma \geq\left(1-\varepsilon^{2}\right)$. Also it is clear that $1-\tau^{2} \leq \varepsilon^{2}$. Therefore, as $\mu^{2}>\sigma^{2}$ we have

(2.33) $\cos 2 t_{1}=\sigma+\mu \geq 2 \sigma \geq\left(1-\varepsilon^{2}\right)$ or $\sin ^{2} 2 t_{1} \leq 1-\left(1-\varepsilon^{2}\right)^{2} \leq 2 \varepsilon^{2}$.

Again since $\mu^{2}=1-\tau^{2}+\sigma^{2} \leq \sigma^{2}+\varepsilon^{2} \leq(\sigma+\varepsilon)^{2}$ we see that $\mu \leq(\sigma+\varepsilon)$ or $\mu-\sigma \leq \varepsilon$. Therefore,

$$
\cos ^{2} 2 t_{2}=(\sigma-\mu)^{2} \leq \varepsilon^{2} \text { or } \sin ^{2} 2 t_{2} \geq\left(1-\varepsilon^{2}\right) .
$$

Hence $\sin 2 t_{2} \geq 1600 \sin 2 t_{1}$ will hold true once we have $\left(1-\varepsilon^{2}\right) \geq 2 \varepsilon^{2}(1600)^{2}$, i.e. if we have $\left\{1+2(1600)^{2}\right\} \varepsilon^{2} \leq 1$ which is true by the choice of $\varepsilon$.

Having proved all the preliminary lemmas we can now complete the proof of Theorem 2.1.

Proof of Theorem 2.1. As we have already remarked we need to show that

$$
\sup _{y} \int\left|F_{n}(x, y)\right| d x \geq C N \text {. }
$$

Looking at just the main term in $\operatorname{Re} F_{n}(x, y)$ we have in view of Lemma 2.3

$$
\left|F_{n}(x, y)\right| \geq c N^{-1 / 2}\left\{\frac{1}{2}\left(\sin 2 t_{1}\right)^{-1}\left|\cos 2 \psi\left(t_{1}\right)\right|-2\left(\sin 2 t_{2}\right)^{-1}\right\} .
$$

Let $E$ denote the set of all $x$ and $y$ satisfying the conditions of Lemma 2.5. Using the results of Lemma 2.5 and Lemma 2.6 we get for at least $2 / 3$ of $N$ in the interval $I$

$$
\left|F_{n}(x, y)\right| \geq 2 c N^{-1 / 2}\left(\sin 2 t_{2}\right)^{-1} \geq 2 c N^{-1 / 2} .
$$

Therefore, for those $N$ we have $\int\left|F_{n}(x, y)\right| d x \geq \int_{E}\left|F_{n}(x, y)\right| d x \geq C N$ since the Lebesgue measure of $E$ is $c N^{3 / 2}$ for some constant $c$. This completes the proof of the theorem. 
Thus we see that in the three-dimensional case the Riesz means of order smaller than 1 are not effective for the $L^{1}$ summability. In the sections to come we will get good estimates for the kernel when the index is bigger than 1 and prove the uniform boundedness of the Riesz means.

\section{Estimation OF THE KeRNEL WHEN $\alpha>(3 n-2) / 6$}

We will estimate the kernel of the Riesz means when $\alpha>(3 n-2) / 6$ in this section. For that purpose we need a good expression for the kernel. We consider $n=3$ for the sake of simplicity. As in the one-dimensional case our starting point is the Mehler kernel. Consider the kernel $G_{r}(t, x, y)$ defined as follows:

$$
G_{r}(t, x, y)=\sum_{k \geq 0} e^{-(2 k+3) i t} r^{k} \Phi_{k}(x, y) .
$$

In view of the Mehler's formula this series can be summed. Let $g(t)$ denote the inverse Fourier transform of the function $h(t)$ defined by $h(t)=(1-|t|)^{\alpha}$ for $|t| \leq 1$ and 0 otherwise. Let $S_{R}(\alpha)(x, y)$ denote the kernel of the Riesz means $S_{R}(\alpha)$. Multiplying (3.1) by $R g(R t)$ and integrating we obtain the following formula:

$$
R \int g(R t) G_{r}(t, x, y) d t=\sum_{K \leq R}(1-K / R)^{\alpha} r^{k} \Phi_{k}(x, y)
$$

where we have put $K=2 k+3$. Thus the kernel $S_{R}(\alpha)(x, y)$ is given as a pointwise limit by

$$
S_{R}(\alpha)(x, y)=\lim _{r \rightarrow 1} R \int g(R t) G_{r}(t, x, y) d t .
$$

Next we are going to integrate by parts and then pass to the limit under the integral sign.

Recalling the definition of $A_{r}(t)$ and $B_{r}(t)$ from the previous section we write (3.3) as

$$
S_{R}(\alpha)(x, y)=\lim _{r \rightarrow 1} \pi^{-3 / 2} R \int g(R t) A_{r}(t)^{-3 / 2} \exp \left\{B_{r}(t)\right\} d t .
$$

With the same notations as before we have the equation

$$
\begin{aligned}
\frac{d}{d t}\left\{A_{r}(t)^{-3 / 2}\left\{B_{r}^{\prime}(t)\right\}^{-1}\right\}= & \left(4 r^{2}\right)^{-1} A_{r}(t)^{-1 / 2} C_{r}(t) E_{r}(t)^{-1} \\
& +b\left(2 r^{2}\right)^{-1} A_{r}(t)^{1 / 2} D_{r}(t) E_{r}(t)^{-2} .
\end{aligned}
$$

Therefore, we can write $S_{R}(\alpha)(x, y)=\lim _{r \rightarrow 1}\left\{L_{r}(x, y)-M_{r}(x, y)+N_{r}(x, y)\right\}$ where $L_{r}(x, y), M_{r}(x, y)$ and $N_{r}(x, y)$ are defined as follows:

$$
\begin{gathered}
L_{r}(x, y)=\pi^{-3 / 2} \int R g(R t) \frac{d}{d t}\left\{A_{r}(t)^{-3 / 2}\left\{B_{r}^{\prime}(t)\right\}^{-1} \exp \left\{B_{r}(t)\right\}\right\} d t \\
M_{r}(x, y)=\pi^{-3 / 2}\left(4 r^{2}\right)^{-1} \int R g(R t) \exp B_{r}(t) A_{r}(t)^{-1 / 2} C_{r}(t) E_{r}(t)^{-1} d t
\end{gathered}
$$




$$
N_{r}(x, y)=\pi^{-3 / 2} b\left(2 r^{2}\right)^{-1} \int R g(R t) \exp B_{r}(t) A_{r}(t)^{1 / 2} D_{r}(t) E_{r}(t)^{-2} d t .
$$

Denote the limits of $L_{r}(x, y), M_{r}(x, y)$ and $N_{r}(x, y)$ as $r$ tends to 1 by $L(x, y), M(x, y)$ and $N(x, y)$ respectively. An easy calculation gives the following with $\varphi(t)=-b \operatorname{cosec} 2 t+\frac{1}{2} a^{2} \cot 2 t$ :

$$
\begin{gathered}
L(x, y)=\pi^{-3 / 2} \int R g(R t) \frac{d}{d t}\left\{(\sin 2 t)^{1 / 2}\left(a^{2}-2 b \cos 2 t\right)^{-1} e^{i \varphi(t)}\right\} d t \\
M(x, y)=\pi^{-3 / 2} \int R g(R t)(\sin 2 t)^{-1 / 2} \cos 2 t\left(a^{2}-2 b \cos 2 t\right)^{-1} e^{i \varphi(t)} d t \\
N(x, y)=\pi^{-3 / 2} \int R g(R t)(\sin 2 t)^{-3 / 2} \varphi^{\prime \prime}(t) \varphi^{\prime}(t)^{-2} e^{i \varphi(t)} d t
\end{gathered}
$$

Lemma 3.1. Assume that $x$ is different from $y$ and $-y$. Then $S_{R}(\alpha)(x, y)$ is given by

$$
S_{R}(\alpha)(x, y)=L(x, y)-M(x, y)+N(x, y) .
$$

Proof. We only need to show that we can pass to the limit under the integral sign. But that is easily done in exactly the same way as we did in the proof of Lemma 2.1.

Finally we need to compute the function $g(t)$. Since $n=3$, we are interested in the convergence of the Riesz means of order $\alpha$ where $\alpha>1$. We have

Lemma 3.2. Assume that $1<\alpha<2$. Then $g$ is a bounded function and for $t>0$ it is given by $g(t)=\sum_{1 \leq i \leq 3} c_{i} g_{i}(t)$ where $g_{1}(t)=t^{-\alpha-1} e^{i t}, g_{2}(t)=$ $t^{-\alpha-1} e^{-i t}, g_{3}(t)=O\left(t^{-2}\right)$.

Proof. The proof is similar to that of the corresponding lemma in [3]. Here we have to integrate by parts twice to make sure that the resulting integral will converge.

Now we are in a position to estimate the Riesz kernel. First we estimate the kernel when $\alpha$ is greater than 7/6. In this case the estimates are obtained more or less in the same way as the estimates for the one-dimensional kernel are obtained. In the process we will see that many terms give estimates which are good even when $\alpha>1$. We will separate out the bad terms and estimate them in the next section. Again the main tools are the method of stationary phase and integration by parts. First we consider the terms $M$ and $N$ which are very easy to estimate.

For the kernel $S_{R}(\alpha)(x, y)$ we have an easy estimate viz. $\left|S_{R}(\alpha)(x, y)\right| \leq$ $C R^{3 / 2}$. This can be proved by induction using the bounds on the one-dimensional Hermite functions. We give a proof of this fact in Lemma 4.5. First let us estimate the integrals $M$ and $N . M(x, y)$ is given by

$$
M(x, y)=R \int g(R t)(\sin 2 t)^{-1 / 2} \cos 2 t\left(a^{2}-2 b \cos 2 t\right)^{-1} e^{i \varphi(t)} d t .
$$


Since the function $g$ is even it is enough to estimate the integral

$$
I(x, y)=R \int_{t \geq 0} g(R t)(\sin 2 t)^{-1 / 2} \cos 2 t\left(a^{2}-2 b \cos 2 t\right)^{-1} e^{i \varphi(t)} d t .
$$

This integral looks very much like the one that appeared in [3]. Using the periodicity of the function $\varphi$ we can easily convince ourselves that we have only to estimate terms of the form

$$
\begin{aligned}
& A_{k}=R \int_{0}^{\pi / 4} g\{R(t+k \pi)\}(\sin 2 t)^{-1 / 2} \cos 2 t e^{i \varphi(t)} d t, \\
& B_{k}=R \int_{0}^{\pi / 4} g\{R(t+k \pi+\pi / 4)\}(\sin 2 t)^{-1 / 2} \cos 2 t e^{i \varphi^{*}(t)} d t,
\end{aligned}
$$

where $\varphi^{*}(t)=b \operatorname{cosec} 2 t+\frac{1}{2} a^{2} \cot 2 t$. The following estimates are easily checked. For $0 \leq t \leq \pi / 4$

$$
\begin{gathered}
-\varphi^{\prime}(t) \sin ^{2} 2 t=a^{2}-2 b \cos 2 t \geq \frac{1}{2}|x-y|^{2}, \\
-\varphi^{* \prime}(t) \sin ^{2} 2 t=a^{2}+2 b \cos 2 t \geq \frac{1}{2}|x+y|^{2}, \\
\left|\varphi^{\prime \prime}(t) \sin 2 t\right| \leq 4\left|\varphi^{\prime}(t)\right|, \\
\left|\varphi^{* \prime \prime}(t) \sin 2 t\right| \leq 4\left|\varphi^{* \prime}(t)\right| .
\end{gathered}
$$

What really matters is the estimation of $A_{0}$ as the other terms are easily estimated. We will now prove the following estimate for $A_{0}$.

Lemma 3.3. There is a constant $C$ independent of $x, y$ and $R$ such that

$$
\left|A_{0}\right| \leq C R^{3 / 2}\left(1+R^{1 / 2}|x-y|\right)^{-7 / 2} .
$$

Proof. For the sake of convenience let us replace $x$ and $y$ by $R^{1 / 2} x$ and $R^{1 / 2} y$ and consider

$$
D_{0}=\int_{0 \leq t \leq \pi / 4} g\{R(t)\}(\sin 2 t)^{-1 / 2} \cos 2 t\left(a^{2}-2 b \cos 2 t\right)^{-1} e^{i R \varphi(t)} d t .
$$

We need to estimate $D_{0}$ only when $|x-y| \geq 1 / R$. We will prove that $\left|D_{0}\right| \leq$ $C R^{-2}|x-y|^{-7 / 2}$. If we combine this with the estimate $\left|S_{R}(\alpha)(x, y)\right| \leq C R^{3 / 2}$ we obtain $\left|D_{0}\right| \leq C R^{3 / 2}(1+R|x-y|)^{-7 / 2}$. Split the integral into two parts viz. $D_{0}=E_{0}+E=\int_{0 \leq t \leq 1 / R}+\int_{1 / R \leq t \leq \pi / 4}$. Integrating by parts,

$$
\left|E_{0}\right| \leq C R^{-2}|x-y|^{-7 / 2} \text {. }
$$

The term $E$ is a sum of three terms corresponding to the three terms in the expression for $g$. We will consider only the term corresponding to $g_{1}$ as the estimation of the other terms are similar. So we consider the integral

$$
E_{1}=R^{-\alpha-1} \int_{R^{-1}}^{\pi / 4} t^{-\alpha-1}(\sin 2 t)^{-1 / 2}\left\{a^{2}-2 b \cos 2 t\right\}^{-1} \cos 2 t e^{i R t} e^{i R \varphi(t)} d t .
$$


For $|x-y| \geq 1 / 2$ we integrate by parts to give the estimate $\left|E_{3}\right| \leq$ $C R^{-2}|x-y|^{-4}$. When $1 / R \leq|x-y| \leq 1 / 2$ we split the integral into two parts as follows. Let $2 B=|x-y|$ and write $E_{1}=\int_{1 / R \leq t \leq B / 2}+\int_{B / 2 \leq t \leq \pi / 4}$. If $\psi(t)=t+\varphi(t)$, we have $\left|\psi^{\prime}(t)\right| \geq c|x-y|^{2}$ for $1 / R \leq t \leq \frac{1}{2} B$. Therefore, an integration by parts gives the estimate $R^{-2}|x-y|^{-7 / 2}$ for the first integral. Integration gives the estimate $R^{-\alpha-1}|x-y|^{-\alpha-5 / 2}$ for the second integral. Since $1 / R \leq|x-y|$ and $\alpha>1$, we get the estimate $R^{-2}|x-y|^{-7 / 2}$. Thus we have shown $\left|E_{1}\right| \leq C R^{-2}|x-y|^{-7 / 2}$. Returning to the original variables completes the proof of the lemma.

For $A_{k}$ we can show that $\left|A_{k}\right| \leq C k^{-\alpha} R^{3 / 2}\left(1+R^{1 / 2}|x-y|\right)^{-7 / 2}$. The estimation of the term $N(x, y)$ is similar. At some point we have to use the estimate

$$
\left|\varphi^{\prime \prime \prime}(t)\right| \leq \operatorname{cosec} 2 t\left|\varphi^{\prime \prime}(t)\right|+\left|\varphi^{\prime}(t)\right|
$$

which can be easily checked. The integrals involving $\varphi^{*}$ give estimates in terms of $x+y$. We can sum all the terms to get the following final estimate for $M$ and $N$.

Proposition 3.1. For some constant $C$ independent of $x, y$ and $R$ we have

$$
|M(x, y)| \leq C\left\{R^{3 / 2}\left(1+R^{1 / 2}|x-y|\right)^{-7 / 2}+R^{3 / 2}\left(1+R^{1 / 2}|x+y|\right)^{-7 / 2}\right\}
$$

under the condition that $\alpha>1$. A similar estimate holds for the integral $N(x, y)$ also.

Let us now turn our attention to the estimation of $L$. Recall that $L$ is given by the integral

$$
L(x, y)=R \int g(R t) \frac{d}{d t}\left\{(\sin 2 t)^{1 / 2}\left(a^{2}-2 b \cos 2 t\right)^{-1} e^{i \varphi(t)}\right\} d t .
$$

Integration by parts reduces $L$ to the following form.

$$
L(x, y)=R^{2} \int g^{\prime}(R t)(\sin 2 t)^{1 / 2}\left(a^{2}-2 b \cos 2 t\right)^{-1} e^{i \varphi(t)} d t .
$$

Since $g$ is an even function it is enough to consider the integral from 0 to infinity. As in the case of $M$ and $N$ we can write the above integral as an infinite sum of terms of the form

$$
\begin{aligned}
& I_{k}(x, y)=R^{2} \int_{0}^{\pi / 4} g^{\prime}\{R(t+k \pi)\}(\sin 2 t)^{1 / 2}\left(a^{2}-2 b \cos 2 t\right)^{-1} e^{i R \varphi(t)} d t \\
& J_{k}(x, y)=R^{2} \int_{0}^{\pi / 4} g\{R(t+k \pi+\pi / 4)\}(\sin 2 t)^{1 / 2}\left(a^{2}-2 b \cos 2 t\right)^{-1} e^{i R \varphi^{*}(t)} d t .
\end{aligned}
$$

$I_{0}$ is the only difficult term to estimate. Other $I_{k}$ are easily estimated. The estimation of $J_{k}$ are similar. The only difference is that the estimates will be in terms of $(x+y)$. We estimate only $I_{0}$. 
Splitting the integral into two parts we have $I_{0}=S_{0}+S$ where $S_{0}=\int_{0 \leq t \leq 1 / R}$ and $S=\int_{1 / R \leq t \leq \pi / 4}$. Integration by parts easily gives the estimate $\left|S_{0}\right| \leq$ $C R^{3 / 2}\left(1+R^{1 / 2}|x-y|\right)^{-7 / 2}$. To estimate $S$ we use the expression for $g(t)$. Since $g(t)=\sum_{1 \leq i \leq 3} c_{i} g_{i}(t)$ we see that $g^{\prime}(t)$ is a sum of many terms. Among all the terms only one term, namely $S_{1}$, is really difficult to estimate. This comes from the term $h_{1}(t)=t^{-\alpha-1} e^{i t}$ of $g^{\prime}(t)$. The contribution of the other terms are somewhat easy to estimate and so we will not consider them. One can easily show that those terms give an estimate of the form $R^{3 / 2}\left(1+R^{1 / 2}|x-y|\right)^{-7 / 2}$. To estimate $S_{1}$ we use the method of stationary phase in the form of the following lemma.

Lemma 3.4 (Van der Corput). Let $\varphi$ be a real valued function and assume that $\left|\varphi^{(\kappa)}(t)\right| \geq c$. When $k=1$ further assume that $\varphi^{\prime}$ is monotonic. Then we have

$$
\left|\int_{[a, b]} \omega(t) e^{i R \varphi(t)} d t\right| \leq C R^{-1 / k}\left\{|\omega(b)|+\int_{[a, b]}\left|\omega^{\prime}(t)\right| d t\right\} .
$$

The estimation of $S_{1}$ is very similar to the estimation of the corresponding integral in the one-dimensional case. So, detailed proofs will be given only if there is a significant difference in the present case. Our aim is to prove the following proposition.

Proposition 3.2. Assume that $7 / 6<\alpha<3 / 2$. Then we have the following estimate for $S_{1}$ :

$$
\left|S_{1}\right| \leq C R^{3 / 2}\left(1+R^{1 / 2}|x-y|\right)^{-\alpha-11 / 6} .
$$

The proof follows in several steps. Recall that the integral $S_{1}$ is given by

$$
S_{1}=R^{-\alpha+1} \int_{R^{-1}}^{\pi / 4} t^{-\alpha-1}(\sin 2 t)^{1 / 2}\left(a^{2}-2 b \cos 2 t\right)^{-1} e^{i R t} e^{i \varphi(t)} d t .
$$

For $|x-y| \leq R^{-1 / 2}$ we use the obvious estimate $C R^{3 / 2}$. For $|x-y| \geq R^{-1 / 2}$ we will prove that $\left|S_{1}\right| \leq C R^{-\alpha / 2+7 / 12}|x-y|^{-\alpha-11 / 6}$. These two estimates will then prove the proposition. Getting the estimate $\left|S_{1}\right| \leq C R^{-\alpha / 2+7 / 12}|x-y|^{-\alpha-11 / 6}$ when $|x-y| \geq 2 \delta$ is easy. Here $\delta$ is any small positive number. As in the one-dimensional case we first show that in the interval $0 \leq t \leq \pi / 4,\left|\psi^{\prime \prime \prime}(t)\right| \geq$ $c|x-y|^{2}$ where $\psi(t)=t+\varphi(t)$. After a preliminary integration by parts we apply Lemma 3.4. That will produce the required estimate.

Estimating $S_{1}$ when $R^{-1 / 2} \leq|x-y| \leq 2 \delta$ is more difficult. Replacing $x$ and $y$ by $R^{1 / 2} x$ and $R^{1 / 2} y$ we consider the integral

$$
K=R^{-\alpha} \int_{R^{-1}}^{\pi / 4} t^{-\alpha-1}(\sin 2 t)^{1 / 2}\left(a^{2}-2 b \cos 2 t\right)^{-1} e^{i R \psi(t)} d t
$$

where $\psi(t)=t+\varphi(t)$. We will prove that $|K| \leq C R^{-\alpha-1 / 3}|x-y|^{-\alpha-11 / 6}$. Let $B=2|x-y|$ and

$$
I=R^{-\alpha} \int_{R^{-1}}^{B / 2} t^{-\alpha-1}(\sin 2 t)^{1 / 2}\left(a^{2}-2 b \cos 2 t\right)^{-1} e^{i R \psi(t)} d t
$$




$$
J=R^{-\alpha} \int_{B / 2}^{\pi / 4} t^{-\alpha-1}(\sin 2 t)^{1 / 2}\left(a^{2}-2 b \cos 2 t\right)^{-1} e^{i R \psi(t)} d t,
$$

so that $K=I+J$. Since $-\psi^{\prime}(t) \sin ^{2} 2 t \geq B^{2}$ for $0 \leq t \leq B / 2$ an integration by parts will give the estimate $|I| \leq C R^{-\alpha-1 / 2}|x-y|^{-\alpha-2}$. To estimate $J$ we rewrite it as

$$
J=-i R^{-\alpha-1} \int_{B / 2}^{\pi / 4} t^{-\alpha-1}(\sin 2 t)^{1 / 2}\left(a^{2}-2 b \cos 2 t\right)^{-1} e^{i R \varphi(t)} d\left\{e^{i R t}\right\} .
$$

Integrating by parts the boundary terms give the estimates $R^{-\alpha-1}|x-y|^{-2}$ and $R^{-\alpha-1}|x-y|^{-\alpha-5 / 2}$. Since $|x-y| \geq R^{-1}$ both estimates are bounded by $R^{-\alpha-1 / 2}|x-y|^{-\alpha-2}$. Many of the differentiated terms give the same estimate. It remains to consider the following term:

$$
J^{\prime}=R^{-\alpha} \int_{B / 2}^{\pi / 4} t^{-\alpha-1}(\sin 2 t)^{-3 / 2} e^{i R \psi(t)} d t .
$$

The estimation of $J^{\prime}$ is easy when $b \leq 0$. Assume that $\delta$ is small enough so that $8 \delta^{2} \leq 1$. Since $|x|^{2}+|y|^{2} \leq|x-y|^{2} \leq 4 \delta^{2} \leq 1 / 2$ there is only one stationary point for the function $\psi$ which is given by $\cos 2 t_{1}=b+m$ where $m^{2}=\left(1-a^{2}+b^{2}\right)$. Observe that $m^{2} \geq 1 / 4$ since $|x|^{2}+|y|^{2} \leq 1 / 2$. When $b>0$ but $|x|^{2}+|y|^{2} \leq 1 / 2$, again there is only one stationary point. The proof of the following lemma is exactly the same as the proof of the corresponding lemma in the one-dimensional case [3] and so it will not be repeated here.

Lemma 3.5. Assume that $b \leq 0$ or $b>0$ with $|x|^{2}+|y|^{2}>1 / 2$. Then for $R^{-1} \leq|x-y| \leq 2 \delta$ we have

$$
\left|J^{\prime}\right| \leq C R^{-\alpha-1 / 2}|x-y|^{-\alpha-2} .
$$

Estimation of $J^{\prime}$ when $b>0$ and $|x|^{2}+|y|^{2}>1 / 2$ is troublesome. We have to treat several cases. First consider the case when $|x|^{2}+|y|^{2}>4$. The estimation of $J^{\prime}$ is easy in this case. We prove the following lemma.

Lemma 3.6. Assume that $b>0$ and $|x|^{2}+|y|^{2}>4$. Then for $R^{-1} \leq|x-y| \leq 2 \delta$ we have

$$
\left|J^{\prime}\right| \leq C R^{-\alpha-1 / 2}|x-y|^{-\alpha-2} \text {. }
$$

Proof. We claim that $\left|\psi^{\prime}(t)\right| \geq 1$. To prove the claim observe that $\psi^{\prime}(t)$ attains a maximum at the point $t_{0}$ where $\psi^{\prime \prime}\left(t_{0}\right)=0$ since $\psi^{\prime \prime \prime}(t) \leq 0$. The point $t_{0}$ is defined by

$$
\cos 2 t_{0}=(|x+y|-|x-y|)(|x+y|+|x-y|)^{-1} .
$$

Let us calculate $\psi^{\prime}\left(t_{0}\right)$. With $\lambda=\cos 2 t_{0}$ we have

$$
-\psi^{\prime}\left(t_{0}\right) \sin ^{2} 2 t_{0}=\lambda^{2}-2 b \lambda+a^{2}-1=\lambda^{-1}\left\{\lambda^{3}-2 b \lambda^{2}+a^{2} \lambda-\lambda\right\} .
$$


Since $\psi^{\prime \prime}\left(t_{0}\right)=0$ we have $a^{2} \lambda-b \lambda^{2}-b=0$ or $a^{2} \lambda=b \lambda^{2}+b$ so that $\left\{\lambda^{3}-2 b \lambda^{2}+a^{2} \lambda-\lambda\right\}=(b-\lambda)\left(1-\lambda^{2}\right)$. Therefore, $\psi^{\prime}\left(t_{0}\right)=1-b \lambda^{-1}$. Since

$$
\lambda^{-1}=(|x+y|-|x-y|)^{-1}(|x+y|+|x-y|)=\frac{1}{4 b}(|x+y|+|x-y|)^{2}
$$

we get $\psi^{\prime}\left(t_{0}\right)=\frac{1}{4}\left\{4-2 a^{2}-2|x-y||x+y|\right\}$. Since $a^{2} \geq 4, \psi^{\prime}\left(t_{0}\right) \leq-1$ or $\left|\psi^{\prime}\left(t_{0}\right)\right| \geq 1$. Hence the claim.

Having proved the claim now it is easy to prove the lemma. Since $\psi^{\prime}(t)$ is monotone in each of the intervals $\frac{1}{2} B \leq t \leq t_{0}$ and $t_{0} \leq t \leq \pi / 4$ we can split $J^{\prime}$ into two parts and apply Lemma 3.4 to obtain the estimate $\left|J^{\prime}\right| \leq$ $C R^{-\alpha-1}|x-y|^{-\alpha-5 / 2}$. Since $|x-y| \geq R^{-1}$ this completes the proof.

Finally we consider the case $1 / 2 \leq|x|^{2}+|y|^{2} \leq 4$. As in the case of one dimension by expanding $\operatorname{cosec} 2 t$ and $\tan t$ in powers of $t$ we can write $\psi(t)=$ $\psi_{1}(t)+\psi_{2}(t)$ where

$$
\begin{gathered}
\psi_{1}(t)=\left\{1+\frac{1}{6}|x-y|^{2}-\frac{1}{2}\left(|x|^{2}+|y|^{2}\right)\right\} t+\frac{1}{4} t^{-1}|x-y|^{2}, \\
\psi_{2}(t)=\frac{1}{2}|x-y|^{2} a(t)-\frac{1}{2}\left(|x|^{2}+|y|^{2}\right) b(t)
\end{gathered}
$$

where $a(t)$ and $b(t)$ are both $O\left(t^{3}\right)$. Again we write $J^{\prime}$ as a sum of two integrals. Let $\rho>0$ be a small number. Define $E$ and $F$ by

$$
\begin{aligned}
& E=R^{-\alpha} \int_{B / 2}^{\rho B^{3 / 5}} t^{-\alpha-1}(\sin 2 t)^{-3 / 2} e^{i R \psi(t)} d t, \\
& F=R^{-\alpha} \int_{\rho B^{3 / 5}}^{\pi / 4} t^{-\alpha-1}(\sin 2 t)^{-3 / 2} e^{i R \psi(t)} d t,
\end{aligned}
$$

so that $J^{\prime}=E+F$. To estimate $E$ we consider two cases. First assume that $1 / 2 \leq|x|^{2}+|y|^{2} \leq 2$. Then taking $\rho=1$ or $\rho=1 / 3 \quad E$ can be estimated as in the one-dimensional case. We have

Lemma 3.7. Assume that $b>0$ and $1 / 2 \leq|x|^{2}+|y|^{2} \leq 2$. Then the estimate

$$
|E| \leq C R^{-\alpha-1 / 2}|x-y|^{-\alpha-2}
$$

is valid for $R^{-1} \leq|x-y| \leq 2 \delta$ provided $\delta$ is chosen sufficiently small.

Next we will estimate $E$ when $2 \leq|x|^{2}+|y|^{2} \leq 4$. For that purpose let us make the following observations. $-\psi^{\prime}(t) \sin ^{2} 2 i=\left(\lambda^{2}-2 b \lambda+a^{2}-1\right)$ is a decreasing function of $\lambda$ for $0 \leq \lambda \leq b$. If $b$ is $>1$ we immediately obtain $-\psi^{\prime}(t) \sin ^{2} 2 t \geq|x-y|^{2}$ for $0 \leq t \leq \pi / 4$. If $b \leq 1$ we will have

$$
-\psi^{\prime}(t) \sin ^{2} 2 t \geq\left(a^{2}-b^{2}-1\right) \text {. }
$$

We claim that $\left(a^{2}-b^{2}-1\right) \geq \frac{1}{2}|x-y|^{2}$. We need to prove that $\left(a^{2}-b^{2}-1\right) \geq$ $\frac{1}{2}\left(a^{2}-2 b\right)$ or $\frac{1}{2} a^{2} \geq\left(1-b+b^{2}\right)$. Since $b \leq 1,\left(1-b+b^{2}\right) \leq 1$ and since $\frac{1}{2} a^{2} \geq 1$ the claim is proved. 
Lemma 3.8. Assume that $b>0$ and $2 \leq|x|^{2}+|y|^{2} \leq 4$. Then the estimate

$$
|E| \leq C R^{-\alpha-1 / 2}|x-y|^{-\alpha-2}
$$

is valid for $R^{-1} \leq|x-y| \leq 2 \delta$ provided $\delta$ is chosen sufficiently small.

Proof. We need to get another estimate for the second derivative of $\psi$. We claim that

$$
\left|\psi^{\prime \prime}(t)\right| \leq 16\left|\psi^{\prime}(t)\right|^{2}|x-y|^{-2} \sin 2 t, \quad \text { for } B / 2 \leq t \leq B^{3 / 5} \text {. }
$$

To prove the claim we recall that $\psi^{\prime \prime}(t)=4 \operatorname{cosec}^{3} 2 t\left(a^{2} \cos 2 t-b \cos ^{2} 2 t-b\right)$ vanishes at the point $t_{0}$ defined by

$$
\cos 2 t_{0}=(|x+y|-|x-y|)(|x+y|+|x-y|)^{-1} \text {. }
$$

First we will show that $\psi^{\prime \prime}(t) \geq 0$ in the interval $0 \leq t \leq B^{3 / 5}$. Since $\left(a^{2} \cos 2 t-b \cos ^{2} 2 t-b\right)$ is decreasing and vanishing at $t_{0}$ it is enough to show that $t_{0} \geq B^{3 / 5}$. As $4 t_{0}^{2} \geq \sin ^{2} 2 t_{0}$, we need to check if $\sin ^{2} 2 t_{0} \geq 4 B^{6 / 5}$. But

$$
\sin ^{2} 2 t_{0}=4|x+y||x-y|(|x+y|+|x-y|)^{-2} \geq|x-y||x+y|^{-1} \geq 2|x-y| \text {. }
$$

Therefore, it is enough to check if $|x-y| \geq 2 B^{6 / 5}$ which is guaranteed if $\delta$ is small enough. Thus $\left(a^{2} \cos 2 t-b \cos ^{2} 2 t-b\right)$ has a maximum of $|x-y|^{2}$ at $t=0$ and therefore, we have the estimate

$$
\left|\psi^{\prime \prime}(t)\right|=4 \operatorname{cosec}^{3} 2 t\left(a^{2} \cos 2 t-b \cos ^{2} 2 t-b\right) \leq 4 \operatorname{cosec}^{3} 2 t|x-y|^{4}|x-y|^{-2} \text {. }
$$

Since $\left|\psi^{\prime}(t) \sin ^{2} 2 t\right| \geq \frac{1}{2}|x-y|^{2}$ we get

$$
\left|\psi^{\prime \prime}(t)\right| \leq 4 \operatorname{cosec}^{3} 2 t|x-y|^{-2} 4\left|\psi^{\prime}(t) \sin ^{2} 2 t\right|^{2}
$$

which proves the claim. Now using the fact that $\left|\psi^{\prime}(t) \sin ^{2} 2 t\right| \geq \frac{1}{2}|x-y|^{2}$ and the estimates on the second derivative an integration by parts will prove the lemma.

Finally the estimation of $F$ is easy. As in the one-dimensional case we can show that for $t$ in the interval $(0, \pi / 4),\left|\psi^{\prime \prime \prime}(t)\right| \geq b$. Since $a \geq 1 / 2$ and $|x-y| \leq 2 \delta$, by choosing $\delta$ small enough we can ensure that $\left|\psi^{\prime \prime \prime}(t)\right| \geq c$ for some constant $c$. Now an application of Lemma 3.4 gives the estimate $|F| \leq C R^{-\alpha-1 / 3}|x-y|^{-3 \alpha / 5-3 / 2}$. Since $|x-y| \leq 2 \delta$, this gives the estimate

$$
|F| \leq C R^{-\alpha-1 / 3}|x-y|^{-\alpha-11 / 6} \text {. }
$$

If we put all the lemmas together and go back to the original variables we get the estimate $R^{-\alpha / 2+7 / 12}|x-y|^{-\alpha-11 / 6}$ for $S_{1}$ as desired. Combining Propositions 3.1 and 3.2 we obtain the following theorem.

Theorem 3.1. Assume that $n=3$. For $\alpha>7 / 6$ we have the following estimate:

$$
\begin{aligned}
& \left|S_{R}(\alpha)(x, y)\right| \leq C\left\{R^{3 / 2}\left(1+R^{1 / 2}|x-y|\right)^{-\alpha-11 / 6}+R^{3 / 2}\left(1+R^{1 / 2}|x-y|\right)^{-7 / 2}\right\} \\
& +C\left\{R^{3 / 2}\left(1+R^{1 / 2}|x+y|\right)^{-\alpha-11 / 6}+R^{3 / 2}\left(1+R^{1 / 2}|x+y|\right)^{-7 / 2}\right\} .
\end{aligned}
$$


If we examine the estimates we see that we are using the condition $\alpha>7 / 6$ only at two places. When $|x-y| \geq R^{1 / 2}$ we use the condition to make sure that the kernel is uniformly integrable. When $R^{-1 / 2} \leq|x-y| \leq R^{1 / 2}$ we obtained the estimate $R^{-\alpha / 2+1 / 2}|x-y|^{-\alpha-2}$ for many terms. These estimates are good enough as long as $\alpha$ is greater than 1. Only in the estimation of $F$ we used the condition $\alpha>7 / 6$. In the next section we will estimate this term when $\alpha>1$. We will also get an estimate for the kernel when $|x-y| \geq R^{1 / 2}$.

\section{Estimation OF THE KeRNEL WHEN $\alpha>(n-1) / 2$}

In this section we will get a good estimate for $S_{R}(\alpha)(x, y)$ when $\alpha>$ $(n-1) / 2$. Recall that we are assuming $n=3$. As we mentioned in the introduction the estimates we are going to get now are not so neat as the estimates we got in the previous section. However, the estimates are sufficient to prove that the Riesz means $S_{R}(\alpha) f$ converge to $f$ in the norm for $\alpha>(n-1) / 2$. The following theorem is the main result of this section.

Theorem 4.1. Assume that $n=3$ and $\alpha>1$. Then the kernel of the Riesz means $S_{R}(\alpha)(x, y)$ is uniformly integrable, i.e. there is a constant $C$ independent of $x, y$ and $R$ such that

$$
\int\left|S_{R}(\alpha)(x, y)\right| d x \leq C \text { and } \int\left|S_{R}(\alpha)(x, y)\right| d y \leq C .
$$

Since $S_{R}(\alpha)(x, y)$ is symmetric in $x$ and $y$ it is enough to prove the first estimate. Replacing $x$ and $y$ by $R^{1 / 2} x$ and $R^{1 / 2} y$ we need to prove that

$$
R^{3 / 2} \int\left|S_{R}(\alpha)\left(R^{1 / 2} x, R^{1 / 2} y\right)\right| d x \leq C .
$$

As we remarked at the end of the last section we have to estimate the integral

$$
F=R^{-\alpha+3 / 2} \int_{\rho B^{3 / 5}}^{\pi / 4} t^{-\alpha-1}(\sin 2 t)^{-3 / 2} e^{i R \psi(t)} d t
$$

for $R^{-1} \leq|x-y| \leq 2 \delta$, where $2 B=|x-y|$. Also we have to estimate the following integral for the range $|x-y| \geq 2 \delta$ :

$$
I=R^{-\alpha+3 / 2} \int_{R^{-1}}^{\pi / 4} t^{-\alpha-1}(\sin 2 t)^{1 / 2}\left(a^{2}-2 b \cos 2 t\right)^{-1} e^{i R \psi(t)} d t .
$$

Since there is rotational symmetry in the kernel we can assume that $y=$ $\left(y_{1}, 0,0\right)$. Also we write $x=\left(x_{1}, x^{\prime}\right)$ where $x^{\prime}$ is in $R^{2}$. Introducing polar coordinates in $R^{2}$, it is enough to show that

$$
\int\left|F\left(x_{1}, y_{1}, r\right)\right| r d x_{1} d r \leq C \text { and } \int\left|I\left(x_{1}, y_{1}, r\right)\right| r d x_{1} d r \leq C
$$

where we have set $r=\left|x^{\prime}\right|$. First we estimate $F$. Recall that we are in the case $x_{1} y_{1} \geq 0$. There are two cases to consider. First we consider the case when either $x_{1}$ or $y_{1}$ exceeds 1 . We prove the following lemma in this case. 
Lemma 4.1. Assume that $x_{1} y_{1}>0,1 / 2 \leq|x|^{2}+|y|^{2} \leq 4$ and either $x_{1}$ or $y_{1}$ exceeds 1 . If $\delta$ is chosen sufficiently small, then for the region $R^{-1} \leq|x-y| \leq$ $2 \delta$ we have the estimate

$$
|F| \leq C\left\{R^{-\alpha+1}|x-y|^{-\alpha-2}+R^{1 / 2}\left(1+R^{1 / 2} r\right)^{-2}\left|x_{1}-y_{1}\right|^{-\alpha / 2-1 / 4} r^{-1}\right\} .
$$

Proof. Let $t_{0}$ be the point where the second derivative of $\psi$ vanishes. As calculated earlier we have $\sin ^{2} 2 t_{0}=4|x+y||x-y|(|x+y|+|x-y|)^{-2}$. From this we obtain

$$
|x-y||x+y|^{-1} \leq \sin ^{2} 2 t_{0} \leq 4|x+y|^{-1}|x-y| .
$$

Since $1 / 2 \leq\left(|x|^{2}+|y|^{2}\right) \leq|x+y|^{2} \leq 2\left(|x|^{2}+|y|^{2}\right) \leq 8$ we see that $c_{1}|x-y|^{1 / 2} \leq$ $\sin 2 t_{0} \leq c_{2}|x-y|^{1 / 2}$. Since $\left|x_{1}-y_{1}\right| \leq \delta$ and $x_{1}$ or $y_{1}$ exceeds 1 we have $x_{1} y_{1} \geq c$ for some $c$ in the region of interest. Therefore, for the third derivative of $\psi$ we have the estimate $\left|\psi^{\prime \prime \prime}(s)\right| \geq x_{1} y_{1} \geq c$. For $t_{0} \leq t \leq \pi / 4, \psi^{\prime \prime}(t)$ is decreasing and we have the estimate

$$
\left|\psi^{\prime \prime}(t)\right|=\int_{t_{0}}^{t}\left|\psi^{\prime \prime \prime}(s)\right| d s \geq c\left(t-t_{0}\right) .
$$

If $2 t_{0} \leq t \leq \pi / 4$, we get $\left|\psi^{\prime \prime}(t)\right| \geq c\left(t-t_{0}\right) \geq c t_{0} \geq c^{\prime}|x-y|^{1 / 2}$. For $B^{3 / 5} \leq t \leq$ $t_{0}, \psi^{\prime \prime}(t)$ is positive and so as above $\psi^{\prime \prime}(t) \geq c\left(t_{0}-t\right)$. If $B^{3 / 5} \leq t \leq \frac{1}{2} t_{0}$, we get $\left|\psi^{\prime \prime}(t)\right| \geq c|x-y|^{1 / 2}$.

Now we can split our integral into three parts. In view of the estimate $\left|\psi^{\prime \prime}(t)\right| \geq c|x-y|^{1 / 2}$ the integrals taken from $B^{3 / 5} \leq t \leq \frac{1}{2} t_{0}$ and $2 t_{0} \leq t \leq \pi / 4$ give the estimates $C R^{-\alpha+1}|x-y|^{-3 \alpha / 5-7 / 4}$ and $C R^{-\alpha+1}|x-y|^{-\alpha / 2-3 / 2}$. Since $|x-y| \leq 2 \delta$ the above are dominated by $C R^{-\alpha+1}|x-y|^{-\alpha-2}$. So it remains to estimate the integral

$$
G=R^{-\alpha+3 / 2} \int_{t_{0} / 2}^{2 t_{0}} t^{-\alpha-1}(\sin 2 t)^{-3 / 2} e^{i R \psi(t)} d t .
$$

To estimate $G$, we have to consider two cases. First of all let us calculate the first derivative of $\psi$. An easy calculation shows that $-\psi^{\prime}(t) \sin ^{2} 2 t=$ $\left\{\lambda^{2}-2 x_{1} y_{1} \lambda+x_{1}^{2}+y_{1}^{2}-1\right\}+r^{2}$. From this it is clear that when $x_{1} y_{1} \geq 1$ we have $-\psi^{\prime}(t) \sin ^{2} 2 t \geq\left(x_{1}-y_{1}\right)^{2}+r^{2}$ and when $x_{1} y_{1}<1$ we have $-\psi^{\prime}(t) \sin ^{2} 2 t \geq$ $-\left(1-x_{1}^{2}\right)\left(1-y_{1}^{2}\right)+r^{2}$. In our present situation as $\left(1-x_{1}^{2}\right)\left(1-y_{1}^{2}\right)$ is negative we have $\left|\psi^{\prime}(t)\right| \geq r^{2}$. An application of Lemma 3.4 will give the following estimate for $G$ :

$$
|G| \leq C R^{-\alpha+1 / 2} r^{-2}|x-y|^{-\alpha / 2-5 / 4} \leq C R^{-1 / 2} r^{-3}\left|x_{1}-y_{1}\right|^{-\alpha / 2-1 / 4} .
$$

We will use this estimate for the range $R^{-1 / 2} \leq r \leq 2 \delta$. When $0 \leq r \leq R^{-1 / 2}$, we use the fact that $\left|\psi^{\prime \prime \prime}(s)\right| \geq x_{1} y_{1} \geq c$ to get the estimate

$$
|G| \leq C R^{-\alpha+3 / 2-1 / 3}|x-y|^{-\alpha / 2-5 / 4} \leq C R^{1 / 2} r^{-1}\left|x_{1}-y_{1}\right|^{-\alpha / 2-1 / 4} .
$$


Combining the two estimates we get

$$
|G| \leq C r^{-1} R^{1 / 2}\left(1+R^{1 / 2} r\right)^{-2}\left|x_{1}-y_{1}\right|^{-\alpha / 2-1 / 4}
$$

and this completes the proof of the lemma.

Next we will estimate $F$ when both $x_{1}$ and $y_{1}$ are less than or equal to 1 . The estimate will be in terms of a function $k_{1}(x, y)$. Let $d^{2}=\left(1-x_{1}^{2}\right)\left(1-y_{1}^{2}\right)$ and define $k_{1}(x, y)$ by

$$
\begin{aligned}
k_{1}(x, y) & =\left(d^{2}-r^{2}\right)^{-1 / 2} r^{-1}, \quad \text { for } 0 \leq r \leq d \\
& =R^{2 / 3}\left\{1+R^{2 / 3}\left(r^{2}-d^{2}\right)\right\}^{-3 / 2}, \text { for } d \leq r \leq 2 \delta \\
& =0, \text { otherwise. }
\end{aligned}
$$

Lemma 4.2. Assume that $x_{1} y_{1}>0,1 / 2 \leq|x|^{2}+|y|^{2} \leq 4$ and both $x_{1}$ and $y_{1}$ are $\leq 1$. Then for the region $R^{-1} \leq|x-y| \leq 2 \delta$ we have the estimate

$$
|F| \leq C\left\{R^{-\alpha+1}|x-y|^{-\alpha-2}+k_{1}(x, y)\left|x_{1}-y_{1}\right|^{-3 \alpha / 5-1 / 4}\right\} \text {. }
$$

Proof. Observe that since both $x_{1}$ and $y_{1}$ are less than or equal to 1 we have $-\psi^{\prime}(t) \sin ^{2} 2 t \geq-d^{2}+r^{2}$. First of all let us consider the case when there are no stationary points, i.e. when $r>d$. For the region $R^{-1} \leq|x-y| \leq R^{-2 / 5}$ we just integrate to get the estimate $|F| \leq C R^{-\alpha+3 / 2}|x-y|^{-3 \alpha / 5-9 / 10}$. Since $R^{1 / 2} \leq|x-y|^{-5 / 4}$ we get

$$
\text { (4.8) }|F| \leq C R^{-\alpha+1}|x-y|^{-3 \alpha / 5-5 / 4-9 / 10} \leq C R^{-\alpha+1}|x-y|^{-\alpha-2}|x-y|^{(8 \alpha-3) / 10} \text {. }
$$

Since $8 \alpha-3 \geq 0$ we obtain the estimate $|F| \leq C R^{-\alpha+1}|x-y|^{-\alpha-2}$. When $R^{-2 / 5} \leq|x-y| \leq 2 \delta$ we proceed as follows. For $r^{2}-d^{2} \geq R^{-2 / 3}$ we apply Lemma 3.4. Since $\left|\psi^{\prime}(t)\right| \geq r^{2}-d^{2}$, we get

$$
\begin{aligned}
|F| & \leq C R^{-\alpha+1 / 2}\left(r^{2}-d^{2}\right)^{-1}|x-y|^{-3 \alpha / 5-3 / 2} \\
& \leq C R^{-\alpha+1 / 2}\left(r^{2}-d^{2}\right)^{-1}\left(\beta^{2}+r^{2}\right)^{-3 \alpha / 10-1 / 8}\left(\beta^{2}+r^{2}\right)^{-5 / 8} \\
& \leq C R^{-\alpha+1 / 2}\left(r^{2}-d^{2}\right)^{-1} \beta^{-3 \alpha / 5-1 / 4}\left(\beta^{2}+r^{2}-d^{2}\right)^{-1 / 2}\left(\beta^{2}+r^{2}\right)^{-1 / 8}
\end{aligned}
$$

where $\beta=\left|x_{1}-y_{1}\right|$. Since $|x-y|^{-1} \leq R^{2 / 5}$ we get the estimate

$$
\begin{aligned}
|F| & \leq C R^{-\alpha+1 / 2+1 / 10}\left(r^{2}-d^{2}\right)^{-3 / 2} \beta^{-3 \alpha / 5-1 / 4} \\
& \leq C R^{-1 / 3}\left(r^{2}-d^{2}\right)^{-3 / 2} \beta^{-3 \alpha / 5-1 / 4}
\end{aligned}
$$

For $0 \leq r^{2}-d^{2} \leq R^{-2 / 3}$ we apply Lemma 3.4 with $k=3$. In view of the estimate $\left|\psi^{\prime \prime \prime}(t)\right| \geq c$ we obtain $|F| \leq C R^{-\alpha+3 / 2-1 / 3}|x-y|^{-3 \alpha / 5-3 / 2}$. Again using $|x-y|^{-1} \leq R^{2 / 5}$ we get the estimate

$$
|F| \leq C R^{-\alpha+3 / 2-1 / 3+1 / 2} \beta^{-3 \alpha / 5-1 / 4} \leq C R^{2 / 3} \beta^{-3 \alpha / 5-1 / 4} .
$$

Combining estimates (4.10) and (4.11) we finally get the estimate

$$
|F| \leq R^{2 / 3}\left\{1+R^{2 / 3}\left(r^{2}-d^{2}\right)\right\}^{-3 / 2} \beta^{-3 \alpha / 5-1 / 4} \text {. }
$$


We will now estimate $F$ when $r \leq d$. Since $|x-y| \leq 2 \delta$, by choosing $\delta$ sufficiently small we can ensure that $x_{1} y_{1} \geq c$ so that $\left|\psi^{\prime \prime \prime}(t)\right| \geq c$. There are two cases to consider. First assume that

$$
m=\left(d^{2}-r^{2}\right)^{1 / 2} \leq R^{-1 / 6}|x-y|^{3 / 10} \text {. }
$$

Using the above estimate $\left|\psi^{\prime \prime \prime}(t)\right| \geq c$ we get

$$
\begin{aligned}
|F| & \leq C R^{-\alpha+3 / 2-1 / 3}|x-y|^{-3 \alpha / 5-3 / 2} \\
& \leq C R^{-\alpha+3 / 2-1 / 3}\left(d^{2}-r^{2}\right)^{-1 / 2} R^{-1 / 6}|x-y|^{3 / 10}|x-y|^{-3 \alpha / 5-3 / 2} \\
& \leq C\left(d^{2}-r^{2}\right)^{-1 / 2} r^{-1}\left|x_{1}-y_{1}\right|^{-3 \alpha / 5-1 / 4} .
\end{aligned}
$$

This takes care of the case when $m \leq R^{-1 / 6}|x-y|^{3 / 10}$.

Next assume that $m>R^{-1 / 6}|x-y|^{3 / 10}$. In this case there are two distinct stationary points given by $\cos 2 t_{1}=b+m$ and $\cos 2 t_{2}=b-m$. Let $t_{0}$ denote the point where $\psi^{\prime \prime}(t)=0$. Without loss of generality we can assume that $B^{3 / 5} \leq t_{1}<t_{0}<t_{2} \leq \pi / 4$. Let $E_{1}=\left(B^{3 / 5}, t_{1}\right), E_{2}=\left(t_{1}, t_{0}\right), E_{3}=$ $\left(t_{0}, t_{2}\right)$ and $E_{4}=\left(t_{2}, \pi / 4\right)$. Let $F_{j}$ be the integral taken over $E_{j}$ so that $F=F_{1}+F_{2}+F_{3}+F_{4}$. We will first estimate $F_{2}$. Since $\psi^{\prime}\left(t_{1}\right)=0$, by Taylor's theorem, we have for $t_{1} \leq t \leq t_{0}$

$$
\psi^{\prime}(t)=\left(t-t_{1}\right) \psi^{\prime \prime}\left(t_{1}\right)+\int_{t_{1}}^{t}(t-s) \psi^{\prime \prime \prime \prime}(s) d s=\left(t-t_{1}\right) \psi^{\prime \prime}(t)+\int_{t_{1}}^{t}\left(s-t_{1}\right)\left(-\psi^{\prime \prime \prime}(s)\right) d s
$$

which gives $\psi^{\prime}(t) \geq\left(t-t_{1}\right) \psi^{\prime \prime}(t)$. As $\psi^{\prime \prime}(t) \geq 0$ for $t_{1} \leq t \leq t_{0}$ we have

$$
\left|\psi^{\prime \prime \prime}(t)\right|=6 \cot 2 t \psi^{\prime \prime}(t)+8 \operatorname{cosec}^{2} 2 t\left(a^{2}-2 b \cos 2 t\right)
$$

and therefore,

$$
\psi^{\prime \prime}\left(t_{1}\right)-\psi^{\prime \prime}(t) \leq\left(t-t_{1}\right)\left\{6 \cot 2 t_{1} \psi^{\prime \prime}\left(t_{1}\right)+8 \operatorname{cosec}^{2} 2 t_{1}\left(a^{2}-2 b \cos 2 t_{1}\right)\right\} .
$$

Since $\psi^{\prime \prime}\left(t_{1}\right)=m \operatorname{cosec} 2 t_{1}$ we get the estimate

$$
\begin{aligned}
\psi^{\prime \prime}\left(t_{1}\right)-\psi^{\prime \prime}(t) & \leq\left(t-t_{1}\right) \operatorname{cosec} 2 t_{1} \psi^{\prime \prime}\left(t_{1}\right)(6+16 / m) \\
& \leq 22(1 / m)\left(t-t_{1}\right) \operatorname{cosec} 2 t_{1} \psi^{\prime \prime}\left(t_{1}\right) .
\end{aligned}
$$

Let $\varepsilon=\frac{1}{44} m \sin 2 t_{1}$. Then for $0 \leq t-t_{1} \leq \varepsilon$, we have $\psi^{\prime \prime}(t) \geq \frac{1}{2} \psi^{\prime \prime}\left(t_{1}\right)$. This also gives for $t \geq t_{1}+\varepsilon, \psi^{\prime}(t) \geq \psi^{\prime}\left(t_{1}+\varepsilon\right) \geq \varepsilon \psi^{\prime \prime}(t) \geq \frac{1}{2} \varepsilon \psi^{\prime \prime}\left(t_{1}\right)$, i.e. $\psi^{\prime}(t) \geq \frac{1}{88} m^{2}$. Splitting the integral into two parts we apply Lemma 3.4 to both of them. For the integral

$$
H=R^{-\alpha+3 / 2} \int_{t_{1}}^{t_{1}+\varepsilon} t^{-\alpha-1}(\sin 2 t)^{-3 / 2} e^{i R \psi(t)} d t
$$

we get the estimate

$$
|H| \leq C R^{-\alpha+1} m^{-1 / 2}\left(\sin 2 t_{1}\right)^{1 / 2}\left\{\left(t_{1}+\varepsilon\right)^{-\alpha-5 / 2}+\int_{t_{1}}^{t_{1}+\varepsilon} t^{-\alpha-7 / 2} d t\right\} .
$$


Since $t_{1} \geq B^{3 / 5}$ the above gives the estimate

$$
\begin{aligned}
|H| & \leq C R^{-\alpha+1} m^{-1}|x-y|^{-3 \alpha / 5-6 / 5} \\
& \leq C\left(d^{2}-r^{2}\right)^{-1 / 2} r^{-1}\left|x_{1}-y_{1}\right|^{-3 \alpha / 5-1 / 4} .
\end{aligned}
$$

Since $\psi^{\prime}(t) \geq \frac{1}{88} m^{2} \geq \frac{1}{88} R^{-1 / 3}|x-y|^{3 / 5}$ in the interval $t_{1}+\varepsilon \leq t \leq t_{0}$, for the remaining part $K$ of $F$ we get the following estimate:

$$
|K| \leq C R^{-\alpha+1 / 2} R^{1 / 3}|x-y|^{-3 \alpha / 5-3 / 2-3 / 5} \leq C R^{-\alpha+1}|x-y|^{-\alpha-2} \text {. }
$$

This takes care of $F_{2}$. The estimation of $F_{1}$ is similar. Next consider the term $F_{3}$. Again we use a Taylor expansion of $\psi^{\prime}$. Since $\psi^{\prime}\left(t_{2}\right)=0$ we have for $t_{0} \leq t \leq t_{2}$

$\psi^{\prime}(t)=\left(t-t_{2}\right) \psi^{\prime \prime}\left(t_{2}\right)+\int_{t_{2}}^{t}(t-s) \psi^{\prime \prime \prime}(s) d s=\left(t_{2}-t\right)\left|\psi^{\prime \prime}(t)\right|+\int_{t}^{t_{2}}\left(t_{2}-s\right)\left(-\psi^{\prime \prime \prime}(s)\right) d s$ which gives $\psi^{\prime}(t) \geq\left(t_{2}-t\right)\left|\psi^{\prime \prime}(t)\right|$. Also we have

$$
\left|\psi^{\prime \prime \prime}(t)\right| \leq 6 \cot 2 t\left|\psi^{\prime \prime}(t)\right|+8 \operatorname{cosec}^{2} 2 t\left(a^{2}-2 b \cos 2 t\right)
$$

and therefore

$$
\psi^{\prime \prime}(t)-\psi^{\prime \prime}\left(t_{2}\right)=\int_{t}^{t_{2}}\left\{-\psi^{\prime \prime \prime}(s)\right\} d s=\int_{t}^{t_{2}}\left\{6 \cot 2 s\left|\psi^{\prime \prime}(s)\right|+16 \operatorname{cosec}^{2} 2 s\right\} d s .
$$

If $\frac{1}{2} t_{2} \leq t \leq t_{2}$ we get

$$
\psi^{\prime \prime}(t)-\psi^{\prime \prime}\left(t_{2}\right) \leq\left(t_{2}-t\right)\left\{6 \operatorname{cosec} t_{2}\left|\psi^{\prime \prime}\left(t_{2}\right)\right|+16 \operatorname{cosec}^{2} t_{2}\right\} .
$$

Since $\operatorname{cosec}^{2} t_{2} \leq 4 \operatorname{cosec}^{2} 2 t_{2}$ this gives

$$
-\left|\psi^{\prime \prime}(t)\right|+\left|\psi^{\prime \prime}\left(t_{2}\right)\right| \leq 76\left(t_{2}-t\right) m^{-1}\left|\psi^{\prime \prime}\left(t_{2}\right)\right| \operatorname{cosec} 2 t_{2} .
$$

If $\varepsilon=\frac{1}{152} m \sin 2 t_{2}$ then for $0 \leq t_{2}-t \leq \varepsilon$, we have $\left|\psi^{\prime \prime}(t)\right| \geq \frac{1}{2}\left|\psi^{\prime \prime}\left(t_{2}\right)\right|=$ $\frac{1}{2} m \operatorname{cosec} 2 t_{2}$. This gives for $t_{0} \leq t \leq t_{1}-\varepsilon$, the bound $\left|\psi^{\prime}(t)\right| \geq \mathrm{cm}^{2} \geq$ $c R^{-1 / 3}|x-y|^{3 / 5}$. Using these observations $F_{3}$ is estimated just like $F_{2}$. The estimation of $F_{4}$ is similar. Putting all the estimates together we complete the proof of the lemma.

This takes care of the term $F$. It is easy to check that

$$
\int_{1 / R \leq|x-y| \leq 2 \delta} F\left(x_{1}, y_{1}, r\right) r d x_{1} d r \leq C .
$$

Therefore, it remains to consider the integral $I$ for the region $|x-y| \geq 2 \delta$. As in the case of $F$ we have to treat two cases, namely when there is no stationary point and when there are stationary points. Again the estimates will be in terms of a function $k(x, y)$ which involves $d$ and $r$. We assume that $1<\alpha \leq 7 / 6$. We first consider the case when $x_{1} y_{1} \geq 1$ or $x_{1} y_{1}<1$ but $\left(1-x_{1}^{2}\right)\left(y_{1}^{2}-1\right)$ is positive. This is the case with no stationary points. We prove the following lemma. 
Lemma 4.3. Assume that $x_{1} y_{1} \geq 1$ or $x_{1} y_{1}<1$ but $\left(1-x_{1}^{2}\right)\left(y_{1}^{2}-1\right) \geq 0$. Then for $|x-y| \geq 2 \delta$ we have

$$
|I(x, y)| \leq C R^{1 / 2}\left(1+R^{1 / 2} r\right)^{-5 / 4}\left(1+\beta^{2}\right)^{-3 / 4} r^{-1} .
$$

Proof. Before going into the proof we recall the following estimate on the second derivative of $\psi$ :

$$
\left|\psi^{\prime \prime}(t)\right| \leq 4\left|\psi^{\prime}(t)\right| \operatorname{cosec} 2 t+4 \operatorname{cosec} 2 t .
$$

As observed earlier $\left|\psi^{\prime}(t)\right| \geq r^{2}$ in the present situation. We have to consider several cases. When $r \geq \delta$ an integration by parts gives the estimate $|I(x, y)| \leq$ $C R^{-\alpha+1 / 2} r^{-2}\left(r^{2}+\beta^{2}\right)^{-1}$. Since $r \geq \delta$ and $\alpha>1$ we can rewrite the above estimate as $|I(x, y)| \leq C R^{-1 / 8} r^{-5 / 4} r^{-1}\left(1+\beta^{2}\right)^{-3 / 4}$. When $\beta \geq \delta$ and $R^{-1 / 2} \leq$ $r \leq \delta$, we split the integral into two parts:

$$
\begin{aligned}
I= & R^{-\alpha+3 / 2} \int_{R^{-1}}^{r^{1 / 2}} t^{-\alpha-1}(\sin 2 t)^{-3 / 2} e^{i R \psi(t)} d t \\
& +R^{-\alpha+3 / 2} \int_{r^{1 / 2}}^{\pi / 4} t^{-\alpha-1}(\sin 2 t)^{-3 / 2} e^{i R \psi(t)} d t .
\end{aligned}
$$

Integration by parts gives the estimate

$$
C R^{-\alpha+1 / 2} r^{-2}\left(r^{2}+\beta^{2}\right)^{-1} r^{-\alpha / 2-1 / 4}
$$

for the first part. Since $\beta \geq \delta$ and $R^{-1 / 2} \leq r$, we get the estimate

$$
C R^{-1 / 8} r^{-5 / 4} r^{-1}\left(1+\beta^{2}\right)^{-3 / 4} \text {. }
$$

Applying Lemma 3.4 to the second part we get the estimate

$$
C R^{-\alpha+1 / 2} r^{-2}\left(r^{2}+\beta^{2}\right)^{-1} r^{-\alpha / 2-1 / 4}
$$

which again is dominated by $R^{-1 / 8} r^{-5 / 4} r^{-1}\left(1+\beta^{2}\right)^{-3 / 4}$. Hence

$$
|I(x, y)| \leq C R^{-1 / 8} r^{-5 / 4} r^{-1}\left(1+\beta^{2}\right)^{-3 / 4}
$$

in the present case also.

When $0 \leq r \leq R^{-1 / 2}$ and $\beta \geq \delta$, we rewrite the integral in the following way. Let us define the function

$$
\sigma(t)=-x_{1} y_{1} \operatorname{cosec} 2 t+\frac{1}{2}\left(x_{1}^{2}+y_{1}^{2}\right) \cot 2 t
$$

and write the integral $I$ as follows:

$$
\begin{aligned}
I=R^{-\alpha+1 / 2} \int_{R^{-1}}^{\pi / 4} & t^{-\alpha-1}(\sin 2 t)^{5 / 2} k(x, y, t) \\
& \times m\left(x_{1}, y_{1}, t\right) e^{i R t} e^{i R r^{2}(\cot 2 t) / 2} d\left\{e^{i R \sigma(t)}\right\}
\end{aligned}
$$

where $k(x, y, t)=\left(a^{2}-2 b \cos 2 t\right)^{-1}$ and $m\left(x_{1}, y_{1}, t\right)=\left(4 x_{1} y_{1} \sin ^{2} t+\beta^{2}\right)^{-1}$. Integrating by parts the boundary terms give the estimate

$$
R^{-\alpha+1 / 2} \beta^{-2}\left(r^{2}+\beta^{2}\right)^{-1} \text {. }
$$


Many of the differentiated terms give the same estimate. When differentiation falls on $e^{i R t}$ we get the estimate $R^{-\alpha+3 / 2} \beta^{-2}\left(r^{2}+\beta^{2}\right)^{-1}$. When differentiation falls on $\exp \left(i R r^{2} \cot 2 t\right)$ we get the same estimate since $r \leq R^{-1 / 2}$ and $\alpha<$ 7/6. Thus we have obtained $|I(x, y)| \leq C R^{-\alpha+3 / 2} \beta^{-2}\left(r^{2}+\beta^{2}\right)^{-1}$. Since $\beta \geq \delta$ and $r \leq R^{-1 / 2}$ we get the estimate

$$
|I(x, y)| \leq C R^{1 / 2} r^{-1}\left(1+\beta^{2}\right)^{-3 / 4}
$$

Thus the following estimates are obtained for $I(x, y)$ :

$$
\begin{aligned}
& |I(x, y)| \leq C R^{1 / 2} r^{-1}\left(1+\beta^{2}\right)^{-3 / 4} \quad \text { for } 0 \leq r \leq R^{-1 / 2}, \\
& \quad|I(x, y)| \leq C R^{-1 / 8} r^{-5 / 4} r^{-1}\left(1+\beta^{2}\right)^{-3 / 4} \text { for } r \geq R^{-1 / 2} .
\end{aligned}
$$

Combining the two estimates we get

$$
|I(x, y)| \leq C R^{1 / 2}\left(1+R^{1 / 2} r\right)^{-5 / 4}\left(1+\beta^{2}\right)^{-3 / 4} r^{-1} .
$$

This completes the proof of the lemma.

Next we want to estimate $I(x, y)$ when $0 \leq x_{1}, y_{1} \leq 1$ still considering the region $|x-y| \geq 2 \delta$. The estimate will be in terms of a function $k_{0}(x, y)$. Let $d^{2}=\left(1-x_{1}^{2}\right)\left(1-y_{1}^{2}\right)$. The function $k_{0}(x, y)$ is defined to be 0 unless $0 \leq x_{1}, y_{1} \leq 1$. When $0 \leq x_{1}, y_{1} \leq 1$ the function is defined by

$$
\begin{aligned}
k_{0}(x, y) & =r^{-4}, \quad \text { for } r \geq 2,0 \leq \beta \leq 1 / 2 \\
& =R^{1 / 2}\left\{1+R^{1 / 2}\left(r^{2}-d^{2}\right)\right\}^{-3 / 2}, \quad \text { for } d \leq r \leq 2, \delta \leq \beta \leq 1 / 2 \\
& =\left(d^{2}-r^{2}\right)^{-1 / 2}, \text { for } 0 \leq r \leq d, \delta \leq \beta \leq 1 / 2 \\
& =R^{1 / 2}\left\{1+R^{1 / 2}\left(r^{2}-d^{2}\right)\right\}^{-3 / 2}, \quad \text { for } d \leq r \leq 2,0 \leq \beta \leq \delta \\
& =\left(d^{2}-r^{2}\right)^{-1 / 2}, \text { for } \delta \leq r \leq d, 0 \leq \beta \leq \delta .
\end{aligned}
$$

We will now prove the following lemma. The proof is somewhat similar to that of Lemma 4.2. Therefore, at times we will make the proof sketchy omitting the details.

Lemma 4.4. Assume that $0 \leq x_{1}, y_{1} \leq 1$ and $|x-y| \geq 2 \delta$. Then we have the estimate

$$
|I(x, y)| \leq C\left\{k_{0}(x, y)+R(1+R|x-y|)^{-3 / 2}|x-y|^{-2}\right\} .
$$

Proof. There are several cases to be treated. First of all observe that $\beta \leq 1 / 2$ since $0 \leq x_{1}, y_{1} \leq 1$. First we assume that $0 \leq r \leq \delta$ and $\delta \leq \beta \leq 1 / 2$ and consider the following integral:

$$
I_{1}=R^{-\alpha+3 / 2} \int_{R^{-1}}^{\beta / 2} t^{-\alpha-1}(\sin 2 t)^{1 / 2}\left(a^{2}-2 b \cos 2 t\right)^{-1} e^{i R \psi(t)} d t .
$$

Since $1 / R \leq t \leq \frac{1}{2} \beta$ in the range of integration we have $\left|\psi^{\prime}(t)\right| \geq \frac{1}{4}|x-y|^{2}$. As $\beta \geq \delta$, and $\alpha>1$ an integration by parts will give the estimate $\left|I_{1}\right| \leq$ 
$C R^{-1 / 2}|x-y|^{-2}$. Since $|x-y| \leq 2$, the above estimate is dominated by $R(1+R|x-y|)^{-3 / 2}|x-y|^{-2}$. Next consider the integral

$$
I_{2}=R^{-\alpha+3 / 2} \int_{\beta / 2}^{\pi / 4} t^{-\alpha-1}(\sin 2 t)^{1 / 2}\left(a^{2}-2 b \cos 2 t\right)^{-1} e^{i R \psi(t)} d t .
$$

Rewriting this integral as

$$
J=-i R^{-\alpha+1 / 2} \int_{\beta / 2 \leq t \leq \pi / 4} t^{-\alpha-1}(\sin 2 t)^{1 / 2}\left(a^{2}-2 b \cos 2 t\right)^{-1} e^{i R \varphi(t)} d\left(e^{i R t}\right)
$$

we observe that the boundary terms are bounded by $R^{-\alpha+1 / 2}|x-y|^{-2}$ which in turn are bounded by $R(1+R|x-y|)^{-3 / 2}|x-y|^{-2}$ as before. When the differentiation falls on the integrand many terms give the same estimate. There remains only one term, viz. the one given by the integral

$$
J=R^{-\alpha+3 / 2} \int_{\beta / 2}^{\pi / 4} t^{-\alpha-1}(\sin 2 t)^{-3 / 2} e^{i R \psi(t)} d t
$$

to be estimated. Now there are two cases to be considered.

First assume that $r>d$. Since $-\psi^{\prime}(t) \sin ^{2} 2 t \geq r^{2}-d^{2}>0$, there are no stationary points in this case. When $r^{2}-d^{2} \geq R^{-1 / 2}$ we can apply Lemma 3.4 to obtain the estimate $C R^{-\alpha+1 / 2}\left(r^{2}-d^{2}\right)^{-3 / 2}$ since $\beta \geq \delta$ and $r \leq 2$. When $r^{2}-d^{2} \leq R^{-1 / 2}$, we can use the following estimate on the third derivative of $\psi:\left|\psi^{\prime \prime \prime}(t)\right| \geq|x-y|^{2} \geq 4 \delta^{2}$. Applying the same lemma we see that $J$ is bounded by a constant times $R^{-\alpha+3 / 2-1 / 3}$ which is $\leq R^{1 / 2}$. Thus we have obtained the estimates

$$
\begin{gathered}
|J| \leq C R^{1 / 2}, \text { for } 0 \leq r^{2}-d^{2} \leq R^{-1 / 2}, \\
|J| \leq C R^{-1 / 4}\left(r^{2}-d^{2}\right)^{-3 / 2}, \text { for } r^{2}-d^{2} \geq R^{-1 / 2} .
\end{gathered}
$$

Combining them we have $|J| \leq C R^{1 / 2}\left\{1+R^{1 / 2}\left(r^{2}-d^{2}\right)\right\}^{-3 / 2}$ when $r>d$. Next assume that $r \leq d$. In this case there are two stationary points in the interval of integration. They are given by $\cos 2 t_{1}=b+m$ and $\cos 2 t_{2}=$ $b-m$ where $m^{2}=d^{2}-r^{2} \geq 0$. Again we have to treat two subcases. When $m \leq R^{-1 / 6}$, we apply Lemma 3.4 with $k=3$. If we use the lower bound $\left|\psi^{\prime \prime \prime}(t)\right| \geq|x-y|^{2} \geq 4 \delta^{2}$ we get the estimate $|J| \leq C R^{-\alpha+3 / 2-1 / 3} \beta^{-\alpha-5 / 2}$. Since $\beta \geq \delta$, and $\alpha \geq 1$ the above gives the estimate $|J| \leq C R^{1 / 6} \leq C\left(d^{2}-r^{2}\right)^{-1 / 2}$. Let us now assume that $m>R^{-1 / 6}$. Without loss of generality we can assume that $\beta / 2 \leq t_{1} \leq t_{2} \leq \pi / 4$.

As in the proof of Lemma 4.2 we can find an $\varepsilon=\mathrm{cm} \sin 2 t_{1}$ such that in an $\varepsilon$ neighbourhood of $t_{1}$ we have $\left|\psi^{\prime \prime}(t)\right| \geq \frac{1}{2} \psi^{\prime \prime}\left(t_{1}\right)=m / 2 \operatorname{cosec} 2 t_{1}$ and outside the $\varepsilon$ neighbourhood the lower bound $\left|\psi^{\prime}(t)\right| \geq m^{2} \geq m^{1 / 2} R^{-1 / 4}$ holds. From these things we obtain

$$
C R^{-\alpha-1} \beta^{-\alpha-5 / 2} m^{-1 / 2} \leq C\left(r^{2}-d^{2}\right)^{-1 / 4}
$$


for the part of the integral taken from $t_{1}$ and $t_{1}+\varepsilon$. For the integral from $t_{1}+\varepsilon$ to $t_{0}$ where $t_{0}$ is the point at which the second derivative vanishes we get the estimate

$$
C R^{-\alpha-3 / 4} \beta^{-\alpha-5 / 2} m^{-1 / 2} \leq C\left(r^{2}-d^{2}\right)^{-1 / 4} .
$$

The integral from $\beta / 2$ to $t_{1}$ gives a similar estimate. We can estimate the integral near $t_{2}$ in a similar fashion. Putting all the estimates together we have $|J| \leq C\left(r^{2}-d^{2}\right)^{1 / 2}$ in the case when $\delta \leq \beta \leq 1 / 2$ and $0 \leq r \leq \delta$.

Next we consider the case when $\delta \leq r \leq 1 / 2$ and $0 \leq \beta \leq \delta$. The estimation is very similar to the previous case. We split the integral at the point $t=r / 4$ and then proceed as before. Finally the case when $r \geq 2$ is easy. Since the first derivative of $\psi$ is bounded away from 0 we immediately get the estimate $|I(x, y)| \leq r^{-4}$. This completes the proof of the lemma.

From Lemmas 4.3 and 4.4 it is easy to see that $I(x, y)$ is uniformly integrable when $\alpha>1$. Thus, we have estimated the kernel in 3 dimensions modulo the estimate $\left|S_{R}(\alpha)(x, y)\right| \leq C R^{3 / 2}$. The estimation in the general case is similar to this. We start with the $n$-dimensional Mehler kernel. Before passing to the limit in $r$ we have to integrate by parts many times. For example, when $n=5$ we integrate by parts twice; when $n=6$ we integrate by parts thrice and so on. The kernel will have many terms and most of them are easily estimated. To estimate the main term we can repeat the arguments of the three-dimensional case. Let us now complete the estimation of the Riesz kernel by proving the following estimate which we have already used.

Lemma 4.5. There is a constant $C>0$ independent of $x, y$ and $R$ such that

$$
\left|S_{R}(\alpha)(x, y)\right| \leq C R^{n / 2} \text {. }
$$

Proof. Recall that the kernel $S_{R}(\alpha)(x, y)$ can be expressed in terms of the kernel $S_{R}(0)=S_{R}$ as given by the following formula:

$$
S_{R}(\alpha)(x, y)=\frac{\Gamma(\alpha+1)}{\Gamma(\alpha)} \int_{0 \leq t \leq 1}(1-t)^{\alpha-1} S_{R t}(x, y) d t
$$

Therefore, any estimate we obtain for the kernel $S_{R}$ will automatically hold true for the kernel $S_{R}(\alpha)(x, y)$ also. So we need to prove $\left|S_{R}(x, y)\right| \leq C R^{n / 2}$. Since $S_{R}(x, y)$ is given by

$$
S_{R}(x, y)=\sum_{0 \leq k \leq R} \Phi_{k}(x, y)
$$

where $\Phi_{k}(x, y)=\sum_{|\nu|=k} \Phi_{\nu}(x) \Phi_{\nu}(y)$ we can actually prove more. Using induction we will prove the following estimate:

$$
\sum_{|\nu| \leq R}\left|\Phi_{\nu}(x)\right|\left|\Phi_{\nu}(y)\right| \leq C R^{n / 2} .
$$


It is enough to prove this estimate for $n=1$. The following estimates for the Hermite functions are well known (see e.g. [25]). There exists positive constants $C$ and $d$ independent of $x$ and $n$ such that for every $n \geq 0$

$$
\begin{aligned}
\left|\varphi_{n}(x)\right| & \leq C\left(N^{1 / 3}+\left|x^{2}-N\right|\right)^{-1 / 4}, \quad \text { if } x^{2} \leq 2 N \\
& \leq C \exp \left(-d x^{2}\right), \quad \text { if } x^{2}>2 N,
\end{aligned}
$$

where as usual $N=2 n+1$. Using these estimates we can easily prove the lemma.

Consider the sum $\sum_{0 \leq k \leq n}\left|\varphi_{k}(x) \varphi_{k}(y)\right|$. Since this is symmetric in $x$ and $y$, we can assume that $x$ and $y$ are both positive and $y<x$. Suppose first that $x^{2} \leq 1$. Then $y^{2} \leq x^{2} \leq K$ where $K=2 k+1$ for all $k=0,1, \ldots, n$. Therefore,

$$
\sum_{0 \leq k \leq n}\left|\varphi_{k}(x) \varphi_{k}(y)\right| \leq C \sum_{0 \leq k \leq n}\left(K^{1 / 3}+\left|x^{2}-K\right|\right)^{-1 / 4}\left(K^{1 / 3}+\left|x^{2}-K\right|\right)^{-1 / 4} .
$$

Applying the Schwarz inequality we get

$$
\begin{aligned}
\sum_{0 \leq k \leq n}\left|\varphi_{k}(x) \varphi_{k}(y)\right| \leq & C\left\{\sum_{0 \leq k \leq n}\left(K^{1 / 3}+\left|x^{2}-K\right|\right)^{-1 / 2}\right\}^{1 / 2} \\
& \times\left\{\sum_{0 \leq k \leq n}\left(K^{1 / 3}+\left|y^{2}-K\right|\right)^{-1 / 2}\right\}^{1 / 2} .
\end{aligned}
$$

Since $K>1$, we have

$$
\begin{aligned}
& \sum_{0 \leq k \leq n}\left(K^{1 / 3}+\left|x^{2}-K\right|\right)^{-1 / 2} \leq \sum_{0 \leq k \leq n}\left(1+\left|x^{2}-K\right|\right)^{-1 / 2} \\
& \quad \leq \sum_{\left|x^{2}-K\right| \leq N}\left(1+\left|x^{2}-K\right|\right)^{-1 / 2} \leq C N^{1 / 2}
\end{aligned}
$$

Therefore, $\sum_{0 \leq k \leq n}\left|\varphi_{k}(x) \varphi_{k}(y)\right| \leq C N^{1 / 2}$.

Getting the estimate when $1<x^{2}<2 N$ is similar. We split the sum into three parts:

$$
\sum_{0 \leq k \leq n}\left|\varphi_{k}(x) \varphi_{k}(y)\right|=\sum_{2 K \leq y^{2}}+\sum_{y^{2} \leq 2 K \leq x^{2}}+\sum_{x^{2} \leq 2 K} .
$$

Each term can be estimated as before using estimates (4.31) without much difficulty. This completes the proof of the lemma.

Using the estimates of $S_{R}(\alpha)(x, y)$ we can prove all three theorems stated in the introduction. When $\alpha>(3 n-2) / 6$ we can obtain the Fejér-Lebesgue and Riemann's localisation theorems. When $\alpha>(n-1) / 2$ we can show that the Riesz means are uniformly bounded on $L^{p}$. Consequently $S_{R}(\alpha) f$ will converge to $f$ in the norm as $R$ tends to infinity. 


\section{REFERENCES}

1. A. Hulanicki and J. W. Jenkins, Almost everywhere summability on nilmanifolds, Trans. Amer. Math. Soc. 278 (1983), 703-715.

2. B. Muckenhoupt, Mean convergence of Hermite and Laguerre series. I, Trans. Amer. Math. Soc. 147 (1970), 419-431.

3. S. Thangavelu, Summability of Hermite expansions. I, Trans. Amer. Math. Soc. 314 (1989), 119-142.

Department of Mathematics, Princeton University, Princeton, New Jersey 08544

Tata Institute of Fundamental Research Centre, Indian Institute of Science Campus, BANGALORE, INDIA 